\title{
1 A highly attenuated Vesiculovax vaccine rapidly protects nonhuman 2 primates against lethal Marburg virus challenge
}

3

4 Courtney Woolsey ${ }^{1,2}$, Robert W. Cross ${ }^{1,2}$, Krystle N. Agans ${ }^{1,2}$, Viktoriya Borisevich ${ }^{1,2}$,

5 Daniel J. Deer ${ }^{1,2}$, Joan B. Geisbert ${ }^{1,2}$, Cheryl Gerardi ${ }^{3}$, Theresa E. Latham ${ }^{3}$, Karla A.

6 Fenton $^{1,2}$, Michael A. Egan ${ }^{4}$, John H. Eldridge ${ }^{4}$, Thomas W. Geisbert ${ }^{1,2 *}$, Demetrius

7 Matassov $^{3 *}$

8

9 Short title: Vesiculovax vaccine rapidly protects against Marburg virus

11 Affiliations:

$12{ }^{1}$ Department of Microbiology and Immunology, University of Texas Medical Branch,

13 Galveston, TX 77555, USA; ${ }^{2}$ Galveston National Laboratory, University of Texas Medical

14 Branch, Galveston, TX 77555, USA; ${ }^{3}$ Department of Viral Vaccine Development, Auro

15 Vaccines, Pearl River, New York 10965, USA; ${ }^{4}$ Department of Immunology, Auro

16 Vaccines, Pearl River, New York 10965, USA.

17

18 *To whom correspondence should be addressed:

19 twgeisbe@utmb.edu

20 dmattasov@aurovaccines.com

22 Keywords: Marburg virus, filovirus, rVSV, VSV, Vesiculovax, ring vaccination,

\section{Ervebo, Angola variant}




\section{Abstract}

25 Background: Marburg virus (MARV), an Ebola-like virus, remains an eminent threat

26 to public health as demonstrated by its high associated mortality rate (23-90\%) and recent

27 emergence in West Africa for the first time. Although a recombinant vesicular stomatitis

28 virus (rVSV)-based vaccine (Ervebo) is licensed for Ebola virus disease (EVD), no

29 approved countermeasures exist against MARV. Results from clinical trials indicate

30 Ervebo prevents EVD in 97.5-100\% of vaccinees 10 days onwards post-immunization.

32 Methodology/Findings: Given the rapid immunogenicity of the Ervebo platform

33 against EVD, we tested whether a similar, but highly attenuated, rVSV-based Vesiculovax

34 vector expressing the glycoprotein (GP) of MARV (rVSV-N4CT1-MARV-GP) could

35 provide swift protection against Marburg virus disease (MVD). Here, groups of

36 cynomolgus monkeys were vaccinated 7,5 , or 3 days before exposure to a lethal dose of

37 MARV (Angola variant). All subjects (100\%) immunized one week prior to challenge

38 survived; $80 \%$ and $20 \%$ of subjects survived when vaccinated 5- and 3-days pre-exposure,

39 respectively. Lethality was associated with higher viral load and aberrant innate immunity

40 signaling, whereas survival correlated with development of MARV GP-specific antibodies

41 and early expression of NK cell-, B-cell-, and cytotoxic T-cell-related transcriptional 42 signatures.

44 Conclusions/Significance: These results emphasize the utility of Vesiculovax 45 vaccines for MVD outbreak management. The highly attenuated nature of rVSV-N4CT1 
46 vaccines, which are clinically safe in humans, may be preferable to vaccines based on the

47 same platform as Ervebo (rVSV “delta G” platform), which in some trial participants

48 induced vaccine-related adverse events in association with viral replication including

49 arthralgia/arthritis, dermatitis, and cutaneous vasculitis.

50 


\section{Author Summary}

52 Marburg virus (MARV) is one of the deadliest viruses known to man. One of the most

53 effective vaccines against this pathogen uses a recombinant vesicular stomatitis virus

54 (rVSV) platform to express MARV glycoprotein (GP) immunogen. As rVSV-based

55 vaccines may be used as medical interventions to mitigate or prevent outbreaks of MARV,

56 defining the time window needed to elicit protection is vital. Here, a rVSV vector

57 expressing MARV glycoprotein (rVSV-N4CT1-MARV-GP) fully protected nonhuman

58 primates from lethality and disease when given as soon as 1 week prior to exposure. At 5-

59 and 3-days pre-exposure, partial protection ( $80 \%$ and $20 \%$ survival, respectively) was

60 achieved. Vaccination with rVSV-N4CT1-MARV-GP appears to "jump-start" the immune

61 system to allow sufficient time for MARV-specific adaptive responses to form. This fast-

62 acting vaccine is based on a similar platform as Ervebo, the only FDA- and EU-approved

63 vaccine for preventing Ebola virus infection. The rVSV-N4CT1-MARV-GP vaccine

64 features additional attenuations in the rVSV backbone that may contribute to a more

65 acceptable safety profile in vaccinees, as Ervebo in some recipients induced vaccine-

66 related adverse events including rashes and joint pain. 


\section{Introduction}

68 The genera Marburgvirus and Ebolavirus are cousins in the family Filoviridae that cause

69 a similar life-threatening hemorrhagic disease in humans and nonhuman primates (NHPs)

70 [1]. Due to their high risk to national security and public health, viruses in both genera are

71 classified as World Health Organization (WHO) High Priority Category A pathogens [2]

72 and US Centers for Disease Control (CDC) Tier 1 select agents [3]. While Ebolavirus

73 contains six genetically distinct species, Marburgvirus contains a single species: Marburg

74 marburgvirus (MARV).

75 In 2004-2005, MARV was responsible for one of the deadliest filovirus outbreaks

76 to date. The virus emerged in the Uige province of Angola resulting in 252 confirmed cases

77 and 227 deaths ( $~ 90 \%$ case fatality rate) [4]. Outbreaks of MARV are primarily restricted

78 to eastern and southern Africa, which largely overlaps with the geographic distribution of

79 its reservoir species, the Egyptian fruit bat (Rousettus aegyptiacus) [5]. While MARV

80 outbreaks have so far been limited and sporadic, field studies in Uganda indicate that 2-

$813 \%$ of Rousette bats are actively infected with Marburgviruses at any given time [6].

82 Biannual seasonal pulses contribute to a $~ 10 \%$ increase in MARV infections in juvenile

83 Rousette bats that coincide with spillover into human populations [6]. This high rate of

84 infection along with the extensive seroprevalence in Rousette bats underscore the

85 underappreciated threat that MARV poses to public health. Marburgviruses have also

86 recently emerged in previously non-endemic regions. On August $6^{\text {th }}, 2021$, the first known

87 case of Marburg virus disease (MVD) in West Africa was reported to the World Health

88 Organization [7]. The case originated in a villager from southwestern Guinea, not far from

89 the Sierra Leonean and Liberian borders. Prior to the outbreak, surveillance in the region 
90 revealed evidence of filoviruses circulating in nearby Sierra Leone, with active MARV

91 infection in approximately $2.5 \%$ of Rousette bats [8]. Virus sequences obtained from

92 Rousette bats in this area were most genetically similar to human isolates identified during

93 the deadly MARV-Angola outbreak (2005), as well as bat isolates in Gabon and the

94 Democratic Republic of Congo (DRC) (2006-2009). This evidence highlights the

95 importance of pathogen surveillance in these regions and stresses the need for medical

96 countermeasures against MVD as spillover events will likely continue to occur.

97 While substantial progress has been made towards the development of vaccines for

98 one ebolavirus species, Zaire ebolavirus (EBOV), no licensed MVD vaccines or

99 therapeutics are currently available. Ervebo® is the only vaccine approved by both the US

100 Food and Drug Administration (FDA) and European Medicines Agency

101 (https://www.ema.europa.eu/en/medicines/human/EPAR/ervebo) and is recommended by

102 the WHO and US Advisory Committee on Immunization Practices (ACIP) for the

103 prevention of Ebola virus disease (EVD) [9, 10]. The vaccine is comprised of a live-

104 attenuated, recombinant vesicular stomatitis virus (rVSV) vector that expresses EBOV

105 glycoprotein (GP) immunogen instead of its native glycoprotein (G). Administration of

106 Ervebo to contacts (and contacts of contacts) of confirmed cases in a ring vaccination trial

107 during the 2013-2016 West Africa and 2018-2020 DRC EBOV outbreaks prevented

108 disease in $97.5-100 \%$ of those immunized within 10 days onwards $[11,12]$. These data

109 demonstrate the ability of Ervebo to serve as a fast-acting vaccine. To protect exposed

110 individuals and reduce community transmission, a similar vaccination strategy could be

111 implemented in the event of a MARV outbreak. 
112 Preclinical studies have demonstrated the use of rVSV vectors to serve as

113 preventative vaccines and postexposure treatments against MVD in guinea pigs and

114 nonhuman primates (NHPs) [13-21]. NHPs serve as the most stringent animal model for

115 testing medical countermeasures and are considered the "gold standard" for recapitulating

116 human manifestations of MVD including coagulopathies [22]. When administered $\sim 1$

117 month before challenge, a single dose of a rVSV vaccine expressing MARV GP fully

118 protected NHPs against a 1000 PFU lethal challenge of various MARV variants [16, 18,

119 20]. Immunity with an rVSV vector was also durable with $100 \%$ protection against a lethal

120 MARV exposure 14 months post-vaccination [23]. Moreover, all NHPs survived when the

121 vaccine was administered 20-30 minutes post MARV (Musoke variant) exposure at the

122 same challenge dose [15]. However, treatment with a rVSV vector at 20-30 minutes after

123 infection failed to fully defend macaques against challenge with the Angola variant of

124 MARV (only 25\% survival) [14]. In vitro studies demonstrate the Angola variant enters

125 host cells via C-type lectin receptors more efficiently than MARV-Musoke, which may

126 contribute to its increased pathogenicity [24]. MARV-Angola also yields a more rapid

127 disease course and a more pronounced pathology in outbred guinea pigs [25] and in NHPs

128 [26], suggesting the Angola variant sets a high bar for MVD vaccines and therapeutics in

129 terms of conferring protection.

130 While preclinical studies have demonstrated the ability of rVSV-based vaccines to

131 rapidly combat Marburgviruses including the Angola variant [13, 14], the exact

132 prophylactic window remains undefined. Recently, Marzi et al. reported that a "delta G"

133 rVSV-based vaccine (rVSV $\Delta$ G-MARV-GP-Angola) similar to Ervebo fully protected

134 NHPs when vaccinated at 7 or 14 days prior to exposure [27]. Partial protection (75\%) was 
135 observed when NHPs were vaccinated 3 days before exposure. Correspondingly,

136 postexposure treatment with a similar vaccine at 20-30 minutes after infection was shown

137 to be $89 \%$ effective in protecting NHPs against a low 50 PFU dose of MARV-Angola [13].

138 Although these results are promising, safety studies with the delta G Ervebo vector have

139 shown undesirable vaccine-related adverse events in humans including prolonged and

140 recurrent arthritis symptoms, maculopapular or vesicular dermatitis, cutaneous vasculitis,

141 and viral shedding [28, 29]. While Ervebo was deemed safe for human use and none of

142 these adverse events were classified as contraindications, a highly effective but less

143 reactogenic next generation vaccine would likely be preferred for widespread

144 immunization.

145 Highly attenuated rVSV-based Vesiculovax vaccines induce low reactogenicity in

146 humans and exhibit fast-acting potential in NHPs [13, 14, 17, 30]. When NHPs were

147 administered rVSV-N2CT1-MARV-GP or rVSV-N4CT1-MARV-GP Vesiculovax

148 vectors 20-30 minutes after a low dose MARV-Angola exposure (50 PFU), survival was

$14980 \%$ and $60 \%$, respectively $[13,14]$. Therefore, Vesiculovax vaccines provide comparable

150 protection as delta $\mathrm{G}$ vaccines. Unlike delta G rVSV vectors, Vesiculovax vectors express

151 target antigen from the first position to increase immunogen expression [17]. The native $\mathrm{G}$

152 is preserved but contains a cytoplasmic tail truncation that interferes with particle

153 maturation due to decreased interaction of $\mathrm{G}$ and the nucleoprotein $(\mathrm{N})$ at the nucleocapsid

154 core [31]. The rVSV-N4CT1-MARV-GP vaccine is a highly attenuated vector containing

155 a rVSV N4 translocation (N2 vectors contain an N2 translocation). Shuffling the rVSV N

156 from the first to the fourth position markedly diminishes the intracellular abundance of this 
157 protein by virtue of its increased distance from the 3 ' transcription promoter [32]. These

158 modifications enable robust attenuation of the vector while retaining high immunogenicity.

159 In this study, we tested the ability of the highly attenuated rVSV-N4CT1-MARV-

160 GP vector to serve as a rapid-acting vaccine for reactive immunization during MVD

161 outbreaks. NHPs were vaccinated at 7, 5, or 3 days before exposure to define the minimum

162 time needed between vaccination and challenge to elicit protective immunity against

163 MARV-Angola. Samples were collected over the course of the study to examine NHPs for

164 clinical signs of disease and to characterize the immune response to vaccination after

165 challenge.

167 Methods

\section{Generation of rVSV vaccine vectors}

169 The rVSV-N4CT1-MARV-GP and rVSVN4CT1-HIVgag vaccine vectors used in this

170 study were recovered from infectious clones as described previously [14, 17]. An

171 expression cassette encoding the full-length MARV-Angola GP (accession number:

172 DQ447653) or HIV gag protein, respectively, was cloned into a plasmid containing the

173 full-length VSV genome. This plasmid encodes for a VSV N1 to N4 gene translocation

174 and VSV G CT1 truncation; the MARV-Angola GP or HIV gag gene is expressed from

175 the first genomic position to maximize GP antigen expression. Vectors were then recovered

176 from Vero cells following electroporation with the resulting plasmids along with VSV

177 helper plasmids. The rescued virus was plaque purified and amplified to produce virus seed

178 stocks. Vaccine vectors were purified and concentrated for in vivo experiments. Before 
179 proceeding to in vivo studies in NHPs, the vector genomes were completely sequenced to

180 verify the fidelity of the open reading frames (ORFs) for all genes.

181

182 Challenge virus

183 The MARV-Angola seed stock originates from the serum of a fatal patient (8-month-old

184 female; isolate 200501379) during the 2004-2005 Uige, Angola outbreak (DQ: 447653.1).

185 The p2 challenge material was created by passaging the original isolate 200501379 twice

186 onto Vero E6 cells (titer $\left.1.5 \times 10^{7} \mathrm{PFU} / \mathrm{mL}\right)$. Stocks were certified free of endotoxin $(<0.5$

$187 \mathrm{EU} / \mathrm{mL}$ ) and mycoplasma contamination.

\section{Ethics Statement}

190 Animal studies were conducted in compliance with the Animal Welfare Act and other

191 federal statutes and regulations relating to animals and experiments involving animals. All

192 experiments adhered to principles stated in the eighth edition of the "Guide for the Care

193 and Use of Laboratory Animals" (National Research Council, 2011). The Galveston

194 National Laboratory (GNL) where this research was conducted (UTMB) is fully accredited

195 by the Association for the Assessment and Accreditation of Laboratory Animal Care

196 International and has an approved OLAW Assurance (\#A3314-01). Animal studies were

197 performed in BSL-4 biocontainment at the University of Texas Medical Branch (UTMB)

198 and the protocol was approved by the UTMB Institutional Biosafety Committee. 


\section{Animal challenge}

201 Eighteen adult (9 females and 9 males) cynomolgus macaques (Macaca fascicularis) of

202 Chinese origin (PreLabs, Worldwide Primates) ranging in age from 3 to 8 years and

203 weighing 2.86 to $7.60 \mathrm{~kg}$ were used for three separate studies at the GNL. Macaques were

204 immunized with a single 10 million PFU intramuscular (i.m.) injection of rVSV-N4CT1-

205 MARV-GP at $7(\mathrm{~N}=5), 5(\mathrm{~N}=5)$, or $3(\mathrm{~N}=5)$ days prior to MARV exposure. Three animals

206 were immunized with an identical dose of rVSVN4CT1-HIVgag at each respective time

207 point to serve as non-specific controls. The inoculation was equally distributed between

208 the left and right quadriceps. All macaques were challenged i.m. in the left quadriceps with

209 a uniformly lethal 1000 PFU target dose of MARV-Angola (actual doses were 1475, 1475,

210 and $1300 \mathrm{PFU}$, respectively). An internal scoring protocol was implemented to track

211 disease progression in challenged animals. Animals were checked at least twice daily for

212 scoring criteria such as posture/activity level, appetite, behavior, respiration, and the

213 presence of hemorrhagic manifestations. Subjects that reached a clinical score $\geq 9$ were

214 promptly euthanized with a pentobarbital solution.

\section{Blood collection}

217 Blood was collected by venipuncture into EDTA and serum tubes pre-challenge and 3, 6, $21810,14,21$, and 28 DPI, or terminally. An aliquot of EDTA-treated whole blood (100 $\mu$ l)

219 was diluted with $600 \mu \mathrm{l}$ of AVL inactivation buffer (Qiagen, Hilden, Germany), and RNA

220 was extracted using a Viral RNA mini-kit (Qiagen) according to the manufacturer's

221 instructions. To isolate plasma and serum, tubes were spun at $2500 \mathrm{rpm}$ for 10 minutes at

$2224^{\circ} \mathrm{C}$. EDTA plasma and serum were stored at $-80^{\circ} \mathrm{C}$ for analysis. 


\section{Hematology and clinical chemistry}

224 Total white blood cell counts, white blood cell differentials, red blood cell counts, platelet

225 counts, hematocrit values, mean cell volumes, mean corpuscular volumes, total

226 hemoglobin concentrations, and mean corpuscular hemoglobin concentrations were

227 analyzed from blood collected in tubes containing EDTA using a laser based hematologic

228 analyzer (VetScan HM5). Serum samples were tested for concentrations of albumin,

229 amylase, alanine aminotransferase (ALT), alkaline phosphatase (ALP), gamma-

230 glutamyltransferase (GGT), aspartate aminotransferase (AST), glucose, total protein,

231 cholesterol, total bilirubin (TBIL), creatine (CRE), blood urea nitrogen (BUN), and C-

232 reactive protein (CRP) by using a Piccolo point-of-care analyzer and Biochemistry Panel

233 Plus analyzer discs (Abaxis).

\section{Viral Load Determination}

236 One-Step Probe RT-qPCR kits (Qiagen) and CFX96 system/software (BioRad) were used

237 to determine viral copies in samples. To detect MARV RNA, we targeted the MARV NP

238 gene with primer pairs and a 6-carboxyfluorescein (6FAM) - $5^{\prime}$ -

239 CCCATAAGGTCACCCTCTT-3' - 6 carboxytetramethylrhodamine (TAMRA) probe.

240 Thermocycler run settings were $50^{\circ} \mathrm{C}$ for $10 \mathrm{~min} ; 95^{\circ} \mathrm{C}$ for $10 \mathrm{~s}$; and 40 cycles of $95^{\circ} \mathrm{C}$

241 for $10 \mathrm{~s}$ plus $59^{\circ} \mathrm{C}$ for $30 \mathrm{~s}$. Integrated DNA Technologies synthesized all primers and Life

242 Technologies customized the probes. Representative MARV genomes were calculated

243 using a genome equivalent standard. The limit of detection for this assay is 1000 copies $/ \mathrm{ml}$. 
245 increasing 10-fold dilutions of plasma samples were adsorbed to Vero E6 monolayers

246 (ATCC Cat: CRL-1586) in duplicate wells $(200 \mu \mathrm{l})$, overlaid with $0.8 \%$ agarose/2x

247 EMEM, and incubated for six days at $37^{\circ} \mathrm{C}$ in $5 \% \mathrm{CO}$. Neutral red stain was added, and

248 plaques were counted after a 24- to 48-hour incubation. The limit of detection for this assay

249 is $25 \mathrm{PFU} / \mathrm{ml}$.

\section{NanoString sample preparation}

252 Targeted transcriptomics was performed on blood samples from macaques as previously

253 described [33]. NHPV2_Immunology reporter and capture probesets (NanoString

254 Technologies) were hybridized with $5 \mu$ l of each RNA sample for $\sim 24$ hours at $65^{\circ} \mathrm{C}$. The

255 RNA:probeset complexes were then loaded onto an nCounter microfluidics cartridge and

256 assayed using a NanoString nCounter® SPRINT Profiler. Samples with an image binding

257 density greater than 2.0 were re-analyzed with $2 \mu$ l of RNA to meet quality control criteria.

\section{Transcriptional analysis}

260 Briefly, nCounter® .RCC files were imported into NanoString nSolver ${ }^{\mathrm{TM}} 4.0$ software. To

261 compensate for varying RNA inputs, an array of housekeeping genes and spiked-in positive

262 and negative controls were used to normalize the raw read counts. The data was analyzed

263 with NanoString nSolver ${ }^{\mathrm{TM}}$ Advanced Analysis 2.0 package to generate principal

264 component (PC) figures, volcano plots, and cell-type trend plots. Human annotations were

265 added for each respective mRNA to perform immune cell profiling within nSolver ${ }^{\mathrm{TM}}$. 
266 Normalized data (fold-change- and p-values) were exported as a .CSV file and imported

267 into GraphPad Prism version 9.3.1 to produce transcript heatmaps. To identify the

268 functional annotation of individual transcripts, we interrogated the GeneCard database

269 (https://www.genecards.org) [34]. For pathway analysis, functional enrichment of

270 normalized counts was performed at the time of challenge, and early, mid, and late disease

271 with Ingenuity Pathway Analysis (Qiagen). Z-scores were imported into GraphPad Prism

272 version 9.3.1 to produce the canonical signaling heatmap.

274 Anti-MARV GP IgM and IgG ELISA

275 MARV GP-specific IgM and IgG antibodies were quantified by ELISA on sera collected

276 at the indicated blood collection days. Immunosorbent MaxiSorp 96-well plates were

277 coated overnight with $15 \mathrm{ng} /$ well $(0.15 \mathrm{~mL})$ of recombinant MARV GPATM $(\Delta \mathrm{TM}$ :

278 transmembrane region absent; Integrated Biotherapeutics, Gaithersburg, MD) in a sodium

279 carbonate/bicarbonate solution ( $\mathrm{pH}$ 9.6). Antigen-adsorbed wells were subsequently

280 blocked with $2 \%$ bovine serum antigen (BSA) in 1 x PBS for at least two hours. Sera were

281 initially diluted 1:100 and then two-fold through 1:12800 in ELISA diluent (2\% BSA in

$2821 \times$ PBS, and $0.2 \%$ Tween-20). After a one-hour incubation, cells were washed four times

283 with wash buffer (1 x PBS with $0.2 \%$ Tween-20) and incubated for an hour with a dilution

284 of horseradish peroxidase (HRP)-conjugated anti-rhesus IgM (1:2500) or IgG antibody

285 (1:5000) (Fitzgerald Industries International, Acton, MA). SigmaFast O-phenylenediamine

286 (OPD) substrate (Sigma; P9187) was added to the wells after four additional washes to

287 develop the colorimetric reaction. The reaction was stopped with $3 \mathrm{M}$ sulfuric acid $\sim 5$

288 minutes after OPD addition and absorbance values were measured at a wavelength of 
$289492 \mathrm{~nm}$ on a Cytek Cytation 5. Absorbance values were determined by subtracting uncoated

290 from antigen-coated wells at the corresponding serum dilution. End-point titers were

291 defined as the reciprocal of the last adjusted serum dilution with a value $\geq 0.20$.

\section{Statistical analysis}

294 Statistical analysis of viral load was carried out in GraphPad Prism version 9.3.1

295 (GraphPad, Software, Inc., La Jolla, CA) using a mixed-effects model with Geisser-

296 Greenhouse correction and a Dunnett's multiple comparisons test. A multiple hypothesis

297 Benjamini-Hochberg false discovery rate (FDR) corrected p-value less than 0.05 was

298 deemed significant for transcriptional analyses, unless otherwise stated. A Pearson

299 correlation coefficient was employed to measure linear correlation between individual

300 subject viremia levels and expression of specific DE transcripts.

\section{Results}

303 To define the prophylactic window afforded by Vesiculovax vaccination against MVD, we

304 immunized 15 cynomolgus macaques with a single 10 million PFU i.m. dose of

305 rVSVN4CT1-MARV-GP at $7(\mathrm{~N}=5), 5(\mathrm{~N}=5)$, or $3(\mathrm{~N}=5)$ days before challenge $($ Fig $\mathbf{1 A})$.

306 Three additional subjects were inoculated with an irrelevant rVSVN4CT1-HIV-gag1

307 vaccine at $7(\mathrm{~N}=1), 5(\mathrm{~N}=1)$, or $3(\mathrm{~N}=1)$ days prior to MARV exposure to control for non-

308 specific effects of the vector. All macaques were i.m. challenged with a uniformly lethal

309 target dose of 1000 PFU of MARV-Angola and observed daily for signs of illness up to

310 the 28 days post-infection (DPI) study endpoint. 
A

rVSV-N4CT1-MARV-GP

Vesiculovax

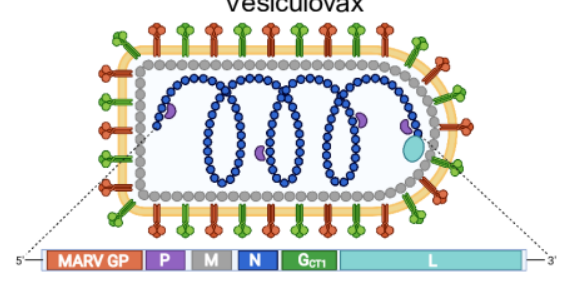

Vector control

Vaccinated
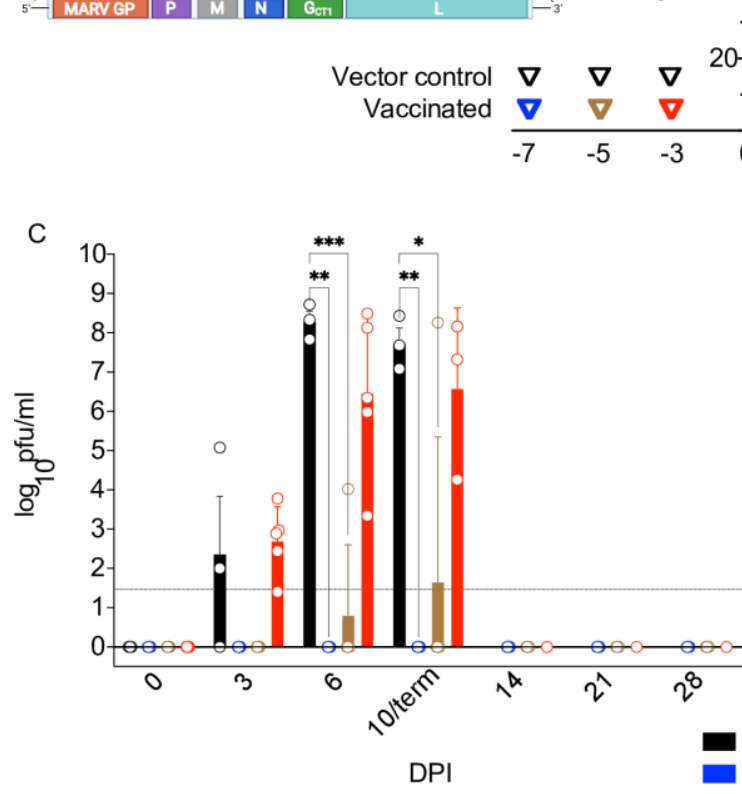

B

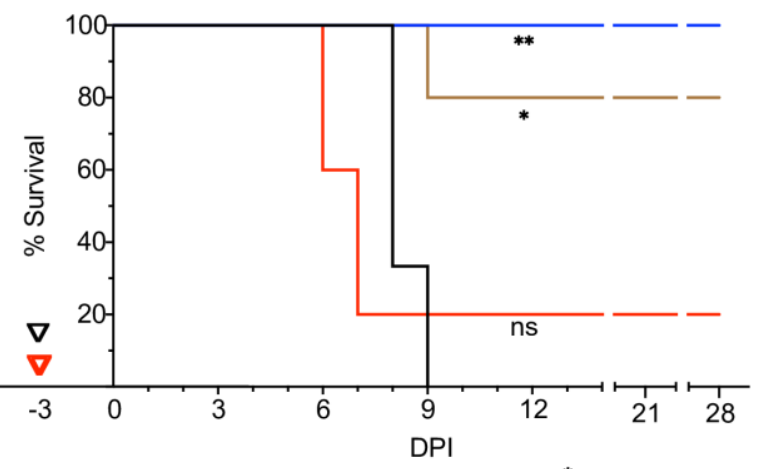

D

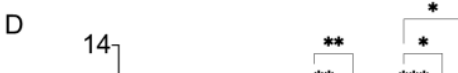

14
12

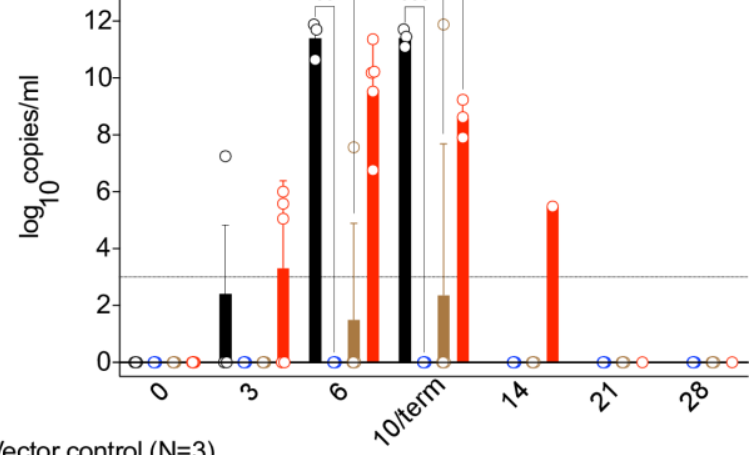

Vector control $(\mathrm{N}=3)$

-7 DPI Vaccinated $(\mathrm{N}=5)$

-5 DPI Vaccinated $(\mathrm{N}=5)$
-3 DPI Vaccinated $(\mathrm{N}=5)$

311

312 Fig 1. Study Design and comparison of viral loads in vaccinated macaques exposed to

\section{MARV-Angola.}

314 (A) rVSV-N4CT1-MARV-GP vector design and genome organization. The Vesiculovax

315 vaccine encodes a truncated form (CT1) of the native VSV G (green box); the N gene was

316 translocated from the $1^{\text {st }}$ position to the 4 th position $(\mathrm{N} 4)$ of the genome (blue box). The

317 MARV GP glycoprotein is expressed from the $1^{\text {st }}$ position to increase immunogen

318 expression (orange box). (B) Survival curves of vaccinated cohorts immunized with rVSV-

319 N4CT1-MARV-GP at -7 DPI (blue; n=5), -5 DPI (brown; n=5), or -3DPI (red; n=5). A

320 single vector control subject was vaccinated at each respective time point (black; $n=3$ ). A

321 log-rank test was used to determine statistical significance. Triangles on the $\mathrm{x}$-axis indicate

322 time of vaccination for each respective cohort. (C) Plasma viremia was measured by 
323 standard plaque assay at the denoted time points and reported as $\log 10 \mathrm{PFU} / \mathrm{ml}$. The limit

324 of detection for this assay is $25 \mathrm{PFU} / \mathrm{ml}$ (indicated by a dotted horizontal line). (D) Viral

325 loads were measured by RT-qPCR in whole blood and reported as $\log 10$ copies/ml at the

326 denoted time points. The limit of detection for this assay is 1000 copies/ml (indicated by a

327 dotted horizontal line). For $(\mathbf{C})$ and $(\mathbf{D})$, the average titer \pm SEM is shown for each group

328 at the denoted time points. Statistical significance was determined using a mixed-effects

329 model with Geisser-Greenhouse correction and Dunnett's multiple comparisons test. Not

330 significant (ns); $\mathrm{p}<0.0332(*) ; \mathrm{p}<0.0021(* *) ; \mathrm{p}<0.0002(* * *)$. Abbreviations: rVSV,

331 recombinant Vesicular stomatitis virus; MARV, Marburg virus; GP, MARV glycoprotein;

332 VSV, Vesicular stomatitis virus; G, VSV glycoprotein; N, VSV nucleoprotein; P, VSV

333 phosphoprotein; M, VSV matrix protein; L, VSV polymerase; DPI, days post vaccination;

334 PFU, plaque-forming units.

335 Vaccination with an irrelevant or antigen-specific vector did not appear to delay the

336 onset of disease (Fig 1B). The time-to-death (TTD) was 8-9 DPI for the vector controls

337 and 6-9 DPI for fatal subjects that were specifically vaccinated (chi square test $\mathrm{p}=0.2229$ ).

338 This window is in line with the typical TTD for this animal model (6-9 DPI, mean of 7.3

339 days) $[17,20,35]$. As expected, animals immunized earlier before challenge had a higher

340 rate of survival. Survival rates of groups immunized with rVSVN4CT1-MARV-GP were

341 significantly different than the vector control group with $100 \%$ (log-rank test, $\mathrm{p}=0.0046$ )

342 and $80 \%(\mathrm{p}=0.0153)$ efficacy for -7 DPI and -5 DPI groups, respectively. No statistical

343 difference $(\mathrm{p}=0.5110)$ was noted for the -3 DPI vaccination group, although a sole subject

$344 \quad(20 \%)$ survived. 
346 typical MVD clinical signs such as fever, anorexia, dyspnea, macular rash, and/or

347 depression (S1-S3 Tables). Specifically vaccinated survivors remained healthy and did not

348 display clinical signs of disease other than anorexia at 5 DPI in one subject in the -7 group

349 (Survivor 4) (S1 Table) and transient anorexia and a mild petechial rash in the sole survivor

350 (Survivor 10) in the -3 group (S3 Table). However, all survivors exhibited various

351 hematological changes over the course of the study. Postmortem gross examination of fatal

352 cases in both specifically and non-specifically vaccinated macaques revealed lesions

353 consistent with MVD including subcutaneous hemorrhage; necrotizing hepatitis

354 (characterized as hepatic pallor with reticulation); splenomegaly; lymphadenitis; and

355 hemorrhagic interstitial pneumonia (characterized as failure to completely collapse and

356 multifocal reddening of the lungs) (data not shown). No significant lesions were detected

357 in examined tissues of vaccinated survivors at the study endpoint.

358 As anticipated, serum levels of liver enzymes and kidney function products

359 indicative of organ damage including alanine aminotransferase (ALT), alkaline

360 phosphatase (ALP), aspartate aminotransferase (AST), gamma-glutamyltransferase

361 (GGT), blood urea nitrogen (BUN), and creatinine (CRE) were elevated in fatal cases (S1-

362 S3 Tables). These changes were also noted in Survivor 10 of the -3 group. Lethality also

363 corresponded with elevated CRP along with lymphopenia, thrombocytopenia, and

364 neutrophilia.

365 Survival correlated with lower viral load (Fig 1C and 1D and S1-3 Tables). Viral

366 titers were assessed in each cohort by performing RT-qPCR amplification of viral RNA

367 (vRNA) and conventional plaque assays. Remarkably, neither infectious MARV nor 
368 vRNA was detected in survivors in the -7 and -5 groups (S1 and S2 Tables), whereas the

369 single specifically vaccinated animal that succumbed (Fatal 1) in the -5 cohort had a viral

370 titer of $4.02 \mathrm{LOG}_{10} \mathrm{PFU} / \mathrm{ml}\left(7.56 \mathrm{LOG}_{10}\right.$ copies/ml) at 6 DPI. In comparison, viral loads

371 in vector controls were 3-5 logs higher at the same time point. Similarly, a lower level of

372 viremia was detected in the single survivor (Survivor 10) of the -3 cohort (Survivor 10)

373 (S3 Table). Viral titers were comparable between fatal animals and controls at end-stage

374 disease $\left(\sim 6-8 \mathrm{LOG}_{10} \mathrm{PFU} / \mathrm{ml}\left(\sim 9-11 \mathrm{LOG}_{10}\right.\right.$ copies/ml $\left.)\right)$.

375 To characterize the immune response to Vesiculovax immunization, we performed

376 targeted transcriptomics on whole blood RNA from MARV-exposed vaccinated AGMs.

377 Spatial visualization of the dataset via principal component analyses (PCA) indicated RNA

378 samples clustered independently of time of vaccination (group), but dimensional separation

379 was observed for disposition (fatal, survivor) and DPI (0, 3, 6, 10/terminal) covariates (Fig

380 2A). Minimal expression changes were detected in samples from surviving and non-

381 surviving subjects on the day of challenge. Thereafter, fatal samples exhibited timepoint-

382 distinct clustering irrespective of whether they were derived from specifically or non-

383 specifically vaccinated subjects, denoting similar transcriptional profiles among these

384 animals. Most survivor samples displayed minimal spatial variation at each DPI regardless

385 of the time of vaccination, suggesting overall expression changes in surviving subjects 386 were modest. 
bioRxiv preprint doi: https://doi.org/10.1101/2022.01.22.477345; this version posted January 23, 2022. The copyright holder for this preprint (which was not certified by peer review) is the author/funder, who has granted bioRxiv a license to display the preprint in perpetuity. It is made available under aCC-BY-NC-ND 4.0 International license.

A

A

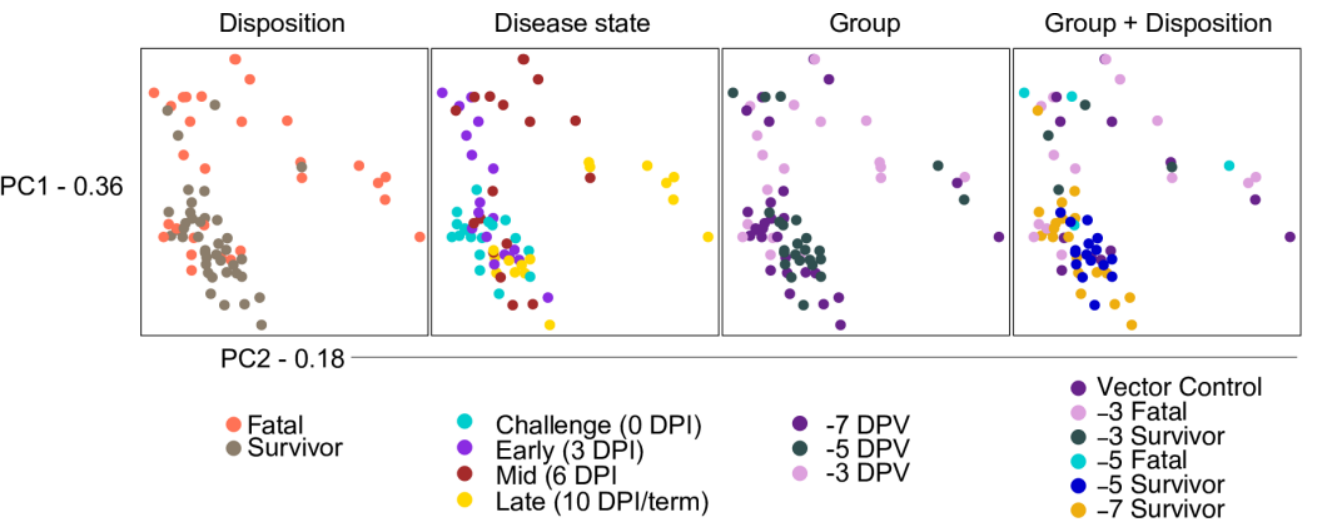

Fig

2.
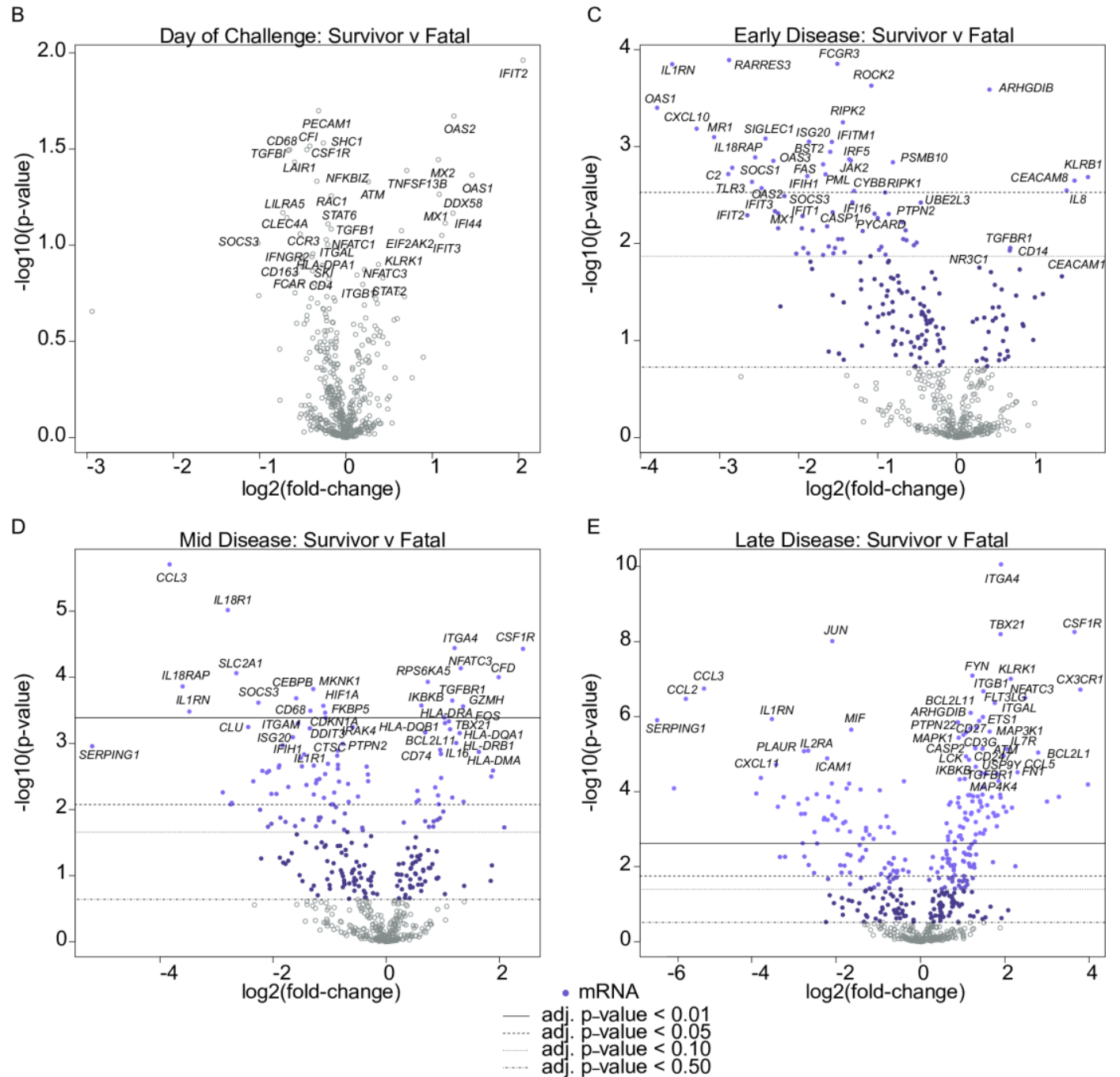

391 Principal component analysis and volcano plots depicting transcriptional changes in

392 vaccinated macaques exposed to MARV-Angola.

393 (A) Shown are principal component (PC) analyses of all normalized transcripts on the day

394 of challenge (0 DPI), and at early (3 DPI), mid (6 DPI), and late disease (10 DPI, or the 
395 terminal time point in fatal cases). Individual samples were filtered by disposition (fatal,

396 survivor), disease state (0, 3, 6, 10/term DPI), group (day of vaccination; -7, -5, or -3 DPI),

397 and group plus disposition (vector control $(n=3),-3$ fatal $(n=4),-3$ survivor $(n=1),-5$ fatal

$398(\mathrm{n}=1),-5$ survivor $(\mathrm{n}=4),-7$ survivor $(\mathrm{n}=5))$. (B) Volcano plots displaying - $\log 10(\mathrm{p}$-values)

399 and $\log 2$ fold changes for each mRNA target on the day of challenge (0 DPI), and at early

400 (3 DPI), mid (6 DPI), and late disease (10 DPI, or the terminal time point in fatal cases).

401 Horizontal lines within the plot indicate adjusted p-value thresholds. Targets highlighted

402 in blue indicate those differentially expressed in the survivor versus fatal group irrespective

403 of time of vaccination or vector administered with a multiple hypothesis Benjamini-

404 Hochberg false discovery rate (FDR) corrected p-value less than 0.05. Abbreviations: PC1

405 (principal component 1); PC2 (principal component 2); DPI, days post infection.

To identify transcriptional signatures of protection, we performed differential 408 expression analysis of survivor and fatal samples at each DPI. Given the lack of significant

409 variation among group (day of vaccination) or vaccine vector administered in fatal cases,

410 we excluded these factors for this analysis. Thus, the survivor dataset included samples

411 from specifically vaccinated survivors at each DPI without respect to time of vaccination,

412 whereas the fatal dataset included samples from both specifically and non-specifically non-

413 surviving subjects at each DPI without respect to time of vaccination.

414 Overall, we identified 76, 70, and 168 differentially expressed (DE) transcripts

415 (Benjamini-Hochberg $(\mathrm{BH})$-adjusted p-value $<0.05)$ in survivor versus fatal samples at

416 early (3 DPI), mid (6 DPI), and late (10 DPI/terminal timepoint) disease, respectively (S1

417 Data). On the day of challenge (0 DPI), survivors tended to express higher levels of 
418 transcripts associated with interferon signaling (e.g., IFIT2, OAS1, OAS2, MX1, MX2, and

419 IFI44) and retinoic acid-inducible gene I (RIG-I; DDX58) signal transduction that is

420 involved in cytosolic detection of double-stranded viral RNA (Fig 2B). These findings

421 suggest earlier activation of innate immunity in these subjects, although no statistically

422 significant difference was found between survivor and fatal samples at this timepoint. At

423 early disease (3 DPI), survivors expressed a higher abundance of natural killer (NK) cell-

424 and cytolytic T lymphocyte (CTL)-affiliated transcripts (KLRB1, KLRD1, GZMK) as well

425 as molecules involved in neutrophil chemotaxis and adhesion (IL-8 and CEACAM 8 ).

426 Conversely, lower expression of inflammatory markers including ILIRN (encodes

427 interleukin 1 receptor antagonist), OAS1 (encodes 2'-5'-oligoadenylate synthetase 1),

428 CXCL10 (encodes C-X-C motif chemokine ligand 10, or IP-10), MRI (encodes major

429 histocompatibility complex, class I-related), RARRES3 (encodes IL-1 receptor antagonist),

430 C2 (encodes complement component C2), IL18RAP (encodes interleukin-18 receptor

431 accessory protein) was evident in survivors (Fig 2C). At mid disease (6 DPI), survivors

432 expressed more adaptive immunity-related transcripts (ITGA4, NFATC3, TGFBR1),

433 antigen presentation-related molecules (HLA-DQB1, HLA-DRA, HLA-DQA1, HLA-DRB1,

$434 H L A-D M A)$, and NK cell-associated transcripts $(G Z M H)$. Also detected in survivors was

435 increased expression of CSF1R (encodes a cytokine that controls the production,

436 differentiation, and function of macrophages) and TBX21 (the master transcriptional

437 regulator for $\mathrm{T}$ helper 1 (Th1) cells). Decreased expression of molecules in survivors

438 included those involved in complement regulation (SERPING1), pro-inflammatory

439 signaling (IL18RAP, IL18R1, ILIRN), metabolism (SLC2A1), or recruitment and activation

440 of monocytes/granulocytes (CCL3, CD68) (Fig 2D). At late disease, again higher 
441 expression of CSF1R, TBX21, and markers of NK cell and T cell activation (CX3CR1,

442 ITGA4, KLRK1, NFATC3, FYN, CD27, CD3G) was noted in survivors, with lower

443 expression of SERPING1, CCL3, ILIRN (Fig 2E).

444 For a more granular assessment, we examined DE transcripts for each group and

445 disposition with respect to a pre-challenge baseline. Samples from specifically vaccinated

446 survivors at -3 DPI and specifically vaccinated fatal subjects at -5 DPI were excluded from

447 this analysis since these cohorts only consisted of a single subject; however, a cursory

448 examination indicated specifically vaccinated survivor versus specifically vaccinated fatal

449 subjects tended to express higher levels of transcripts mapping to the adaptive immunity

450 gene set when vaccinated at 5 versus 3 days prior to challenge (S1 Fig). The topmost

451 upregulated and downregulated DE transcripts in subjects vaccinated with rVSV-N4CT1-

452 MARV-GP at-7 DPI is depicted in Fig. 3. Notably, a similar transcriptional landscape was

453 observed in survivors immunized at -5 or -7 DPI regardless of DPI in line with our PCA

454 results (Fig 3A). Vector controls and non-surviving AGMs vaccinated at -3 DPI also

455 exhibited similar expression profiles. Abundant expression of cytoplasmic granules such

456 as granzymes $(G Z M A, G Z M B, G Z M K)$ and perforin $(P R F 1)$ was apparent in survivors at

457 all time points, suggesting early and sustained activation of NK- and cytotoxic T-cells,

458 whereas granzyme and perforin expression in fatal subjects was not detected until mid or

459 late disease. Th1-associated Tbet (TBX21) was also upregulated in the Survivor -5 and

460 Survivor -7 datasets, whereas this transcription factor was downregulated at all time points

461 in the Fatal -3 cohort and at mid- and late disease in the vector control group. In contrast,

462 repressed transcripts in specifically vaccinated survivors at late disease were implicated in

463 monocyte recruitment (CCRI); pattern recognition receptor signaling (TLR5, MYD88); and 
464 inflammation (TNFAIP6, NFKB1A) (Fig 3B). Thus, survivor transcriptional signatures

465 correlated with predicted Th1 differentiation and activation of NK cell and adaptive

466 responses, whereas lethality correlated with sustained innate immunity signaling and

467 inflammation.

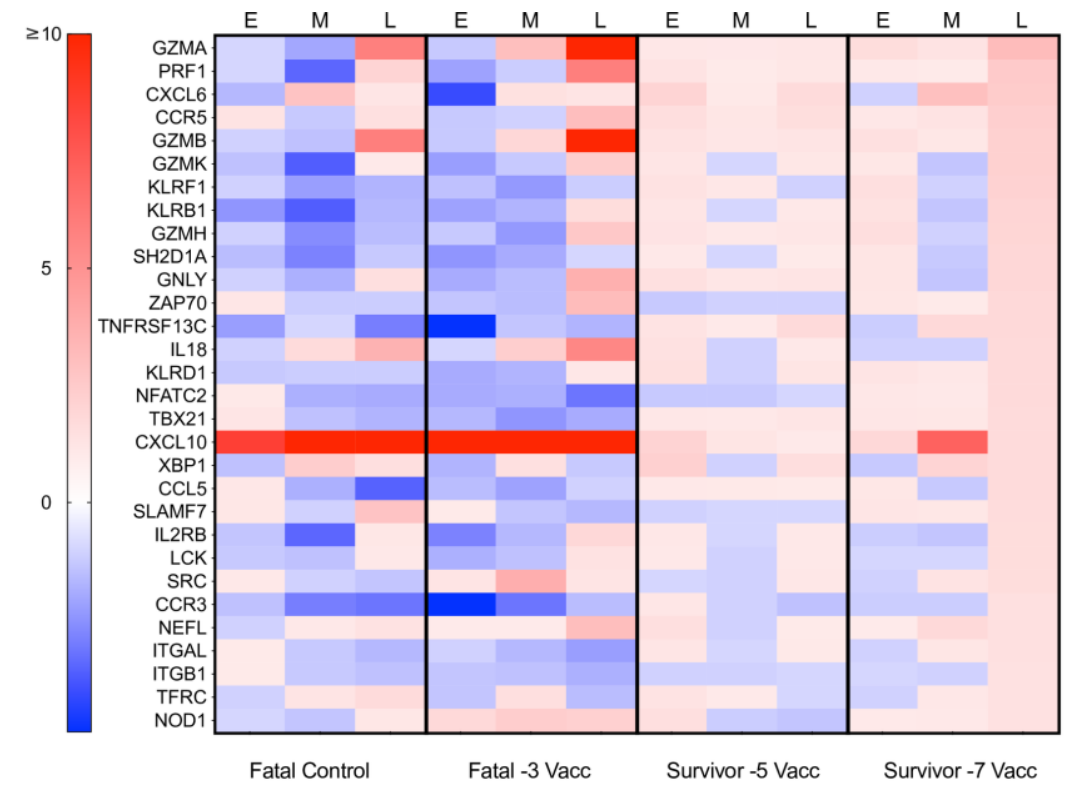

B

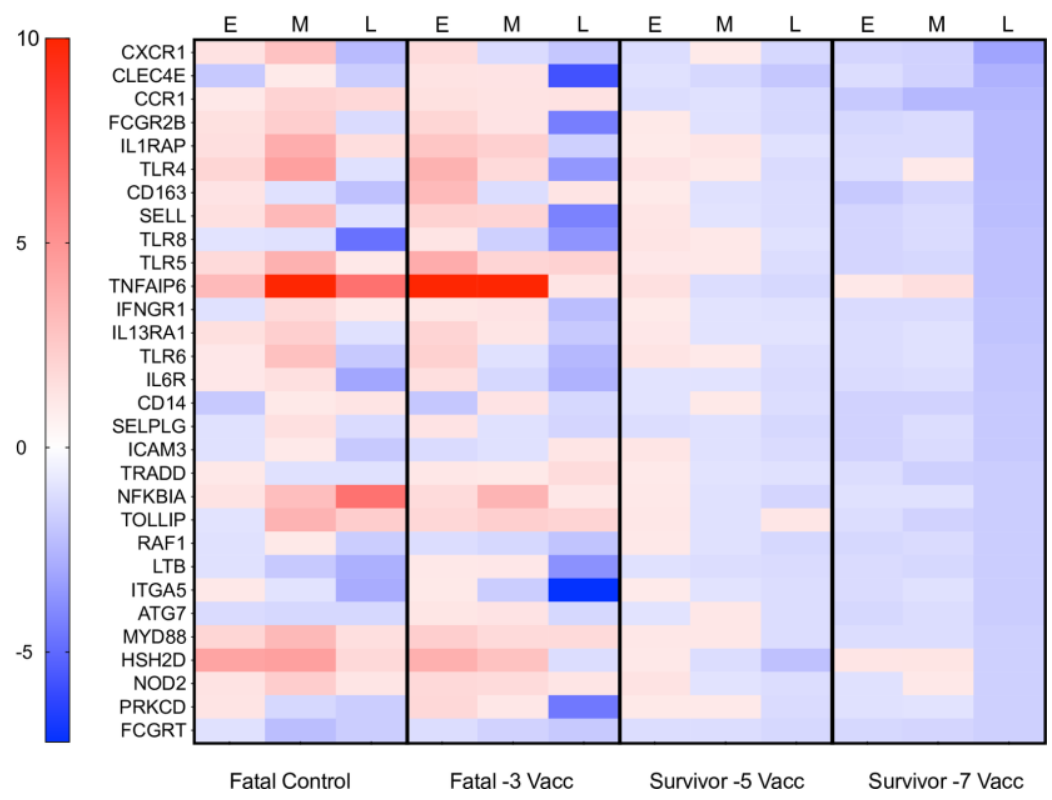

469 Fig 3. Heatmaps depicting the most differentially expressed transcripts in

470 Vesiculovax-vaccinated survivor samples. 
471 The topmost upregulated (A) and downregulated (B) transcripts in vaccinated survivors

472 immunized -7 DPI at late disease. Shown are expression changes in survivor cohorts

473 (survivor $-7 \operatorname{vacc}(n=5)$; survivor $-5 \operatorname{vacc}(n=4))$ and fatal groups (fatal vector control $(n=3)$;

474 fatal $-3 \operatorname{vacc}(n=4))$ at each disease state $(0,3,6,10$ DPI, or the terminal time point in fatal

475 subjects) with respect to a pre-vaccination baseline. A Benjamini-Hochberg false discovery

476 rate (FDR) corrected p-value less than 0.05 was deemed significant. Red indicates high

477 expression; blue indicates low expression; white indicates no change in expression.

478 Abbreviations: Vacc, vaccinated; E, early disease (3 DPI); M, mid disease (6 DPI); L, late

479 disease (10 DPI or terminal timepoint).

480 Fatal outcome was associated with dramatic transcription of genes previously

481 correlated with filovirus disease lethality. These transcripts included pro-inflammatory IP-

48210 (CXCL10; up to a 65- and 55-fold-increase in vector controls and fatal subjects,

483 respectively) [36, 37]; neutrophil-associated calgranulin A (S100A8; up to an 18- and 20-

484 fold-increase in vector controls and fatal subjects, respectively) and calgranulin B (

485 S100A9; up to a 16- and 14-fold-increase in vector controls and fatal subjects, respectively)

486 [33]; and molecules involved in immune exhaustion and anergy (LAG3, CTLA4) [38] (S2A

487 Fig). Positive correlations were found between MARV viremia levels and S100A8

488 (Pearson, $\mathrm{P}<0.0001$ ), S100A9 (Pearson, $\mathrm{P}=0.0001)$, LAG3 (Pearson, $\mathrm{P}=0.0021$ ), and

489 CTLA4 (Pearson, $\mathrm{P}=0.0005)$ counts (S3 Fig). Markedly diminished expression of mRNAs

490 encoding complement factor D (CFD; up to a 32- and 38-fold-decrease) was observed in

491 vector controls and fatal subjects, respectively (S2B Fig).

492 To capture shifts in circulating cell populations associated with survival, we

493 conducted nSolver-based immune cell type profiling at 0, 3, 6, and 10 DPI (or the terminal 
494 time point in euthanized subjects). In agreement with our differential expression results,

495 survival was associated with higher frequencies of cytotoxic cells, Th1 cells, T cells, and

496 B cells (Fig 4). Lower predicted frequencies of neutrophils and macrophages were detected

497 in fatal samples at all time points, confirming our hematology results. Contradicting our

498 DE analysis, decreased frequencies of CD56 ${ }^{\text {dim }}$ NK subsets were found in vaccinated

499 survivors at certain time points. This discrepancy could reflect species-specific

500 immunogenetic differences that exist between human and macaques $[39,40]$ as the nSolver

501 profiling algorithm relies on human NK cell annotations for cell type profiling.
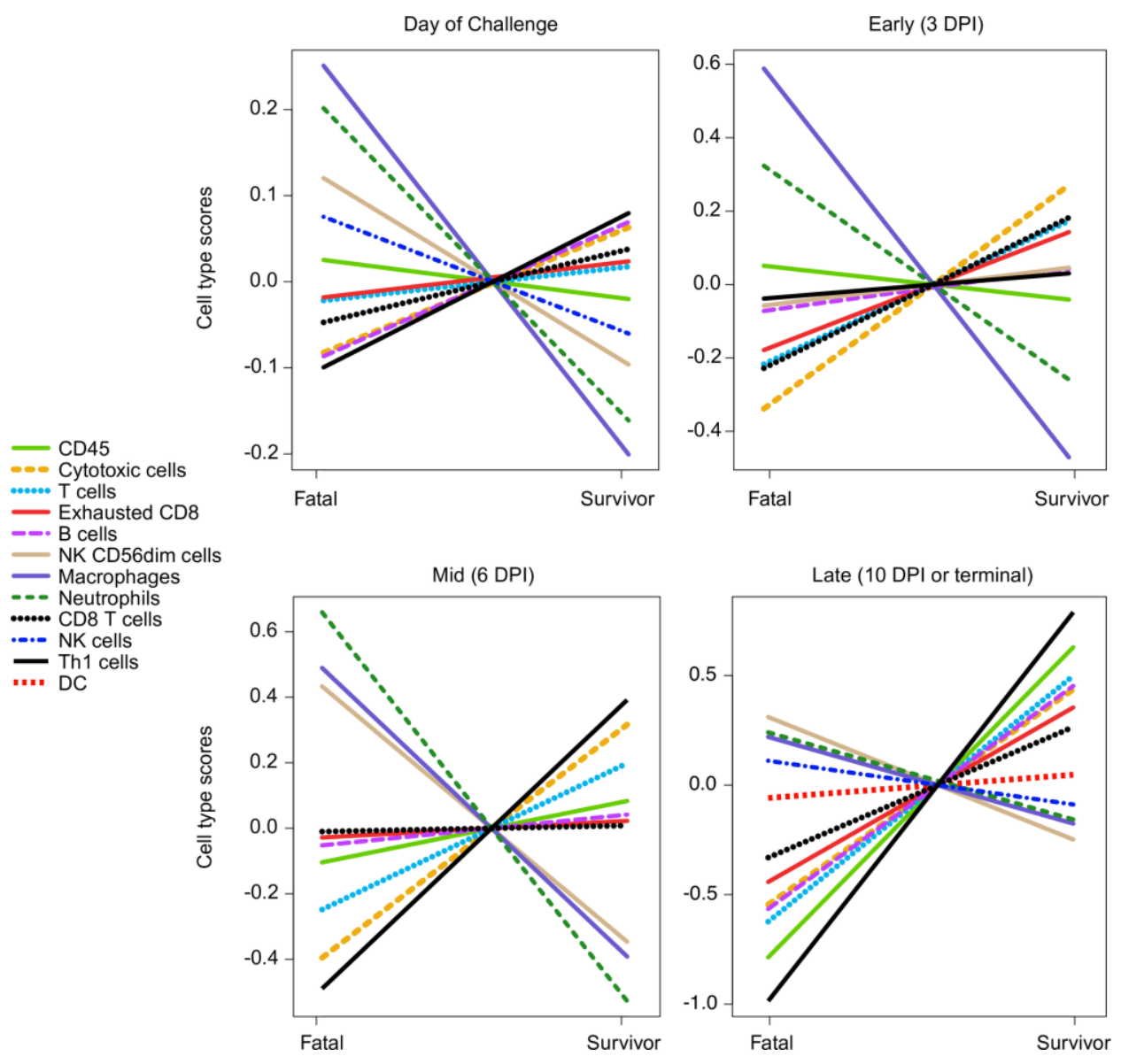

Fig 4. Immune cell type profiling of Vesiculovax-vaccinated survivor and fatal

\section{4 samples.}


505 Respective cell-type quantities for vaccinated survivor $(n=10)$ and fatal $(n=8)$ cohorts on

506 the day of challenge (0 DPI), and early (3 DPI), mid (6 DPI), and late disease (10 DPI, or

507 the terminal time point in fatal cases). A Benjamini-Hochberg false discovery rate (FDR)

508 corrected p-value less than 0.05 was deemed significant for immune cell type profiling of

509 transcripts. DPI, days post infection.

510 Next, enrichment of DE transcripts was executed to unravel canonical signaling

511 pathways associated with rVSV-N4CT1-MARV-GP-elicited protection. At mid and late

512 disease, the top upregulated pathways in survivor versus fatal cases included "PKC-theta

513 signaling in T lymphocytes", "calcium-induced T lymphocyte apoptosis", "iCOS-iCOSL

514 signaling in T helper cells", "CD28 signaling in T-cell helper cells", and "CD27 signaling

515 in lymphocytes" (Fig 5). Thus, positive z-scores in survivors were associated with T cell

516 activation and differentiation of antigen-specific T-cells. Downregulated pathways in

517 survivors at these timepoints included those involved in innate immunity signaling ("role

518 of pattern recognition receptors in recognition of bacteria and viruses", "interferon

519 signaling", "activation of IRF by cytosolic pattern recognition receptors") or immune

520 dysregulation ("systemic lupus erythematous in B cell signaling pathway", "PD-1, PDL-1

521 cancer immunotherapy"). 


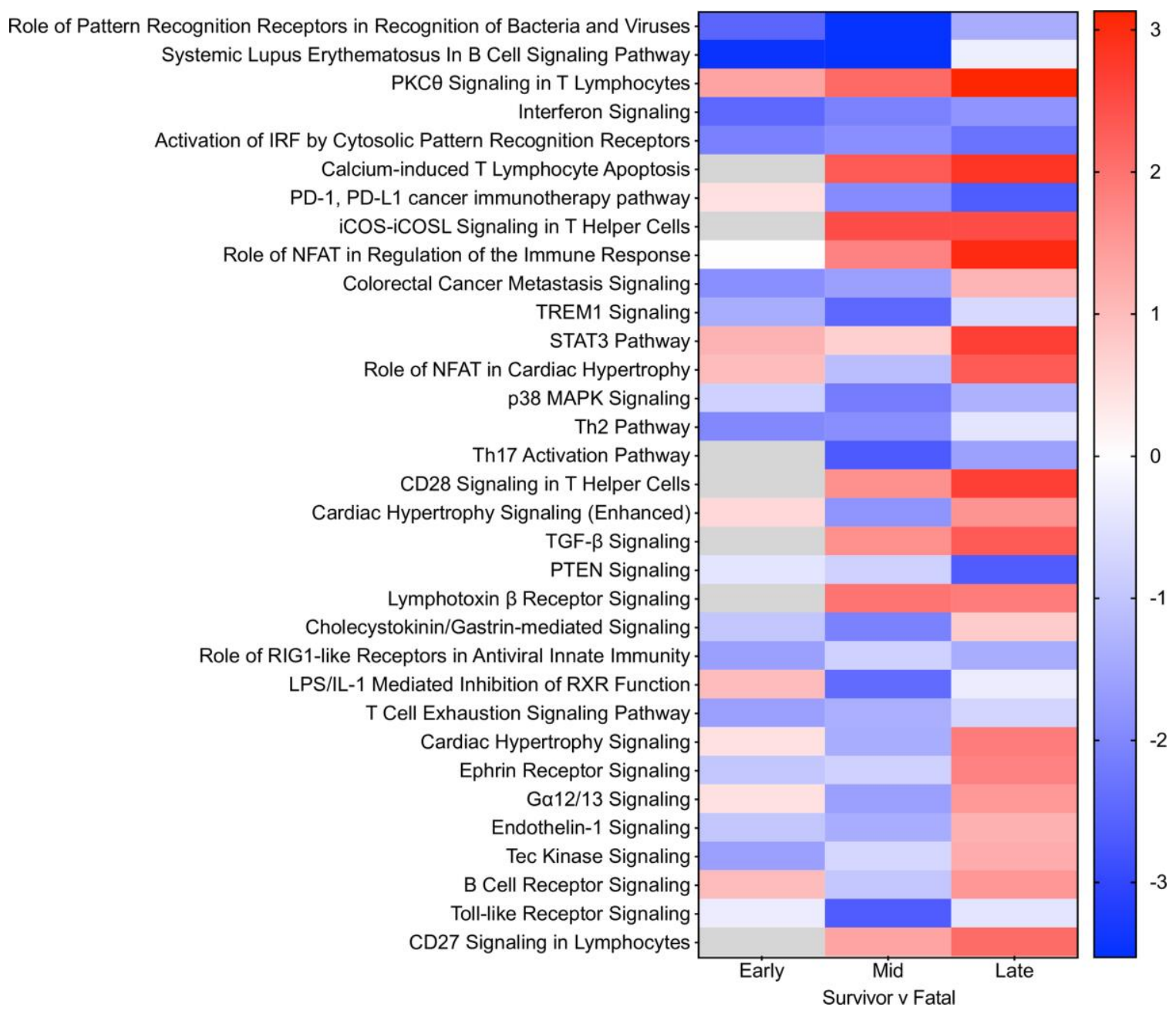

\section{Fig 5. Differentially expressed canonical signaling pathways in Vesiculovax-}

\section{4 vaccinated macaques.}

525 Heatmap of the most significantly upregulated and downregulated canonical pathways in

526 survivor versus fatal subjects based on functional enrichment of differentially expressed

527 transcripts (Benjamini-Hochberg false discovery rate (FDR) corrected p-value less than

$5280.05)$ in vaccinated survivor $(n=10)$ versus fatal $(n=8)$ samples. Cohorts were examined at

529 early (3 DPI), mid (6 DPI), and late disease (10 DPI, or the terminal time point in fatal

530 cases). DPI, days post infection. Corresponding z-scores were plotted. Red indicates high 
531 expression; blue indicates low expression; white indicates no difference in expression; gray

532 indicates insufficient transcripts mapping to the pathway.

533 Lastly, we measured serum IgG and IgM levels in vaccinated macaques to define

534 the contribution of the humoral response to rVSV-N4CT1-MARV-GP-elicited protection.

535 Only specifically vaccinated survivors formed substantial MARV-GP-specific IgG titers,

536 with earlier detection of both immunoglobulin classes in subjects immunized 7 days prior

537 to challenge (as early as the day of challenge in one subject) (Fig 6A and 6B). All surviving

538 subjects had detectable IgG titers by 6 DPI, whereas no evidence of IgG was evident in

539 vector control or non-surviving vaccinated macaques. Low IgM titers (1:100 to 1:800)

540 generally declined during the convalescent stage conjointly with increasing moderate to

541 high titers of $\operatorname{IgG}(1: 400$ to $1: 12,800)$ (Fig 6B).

542

A

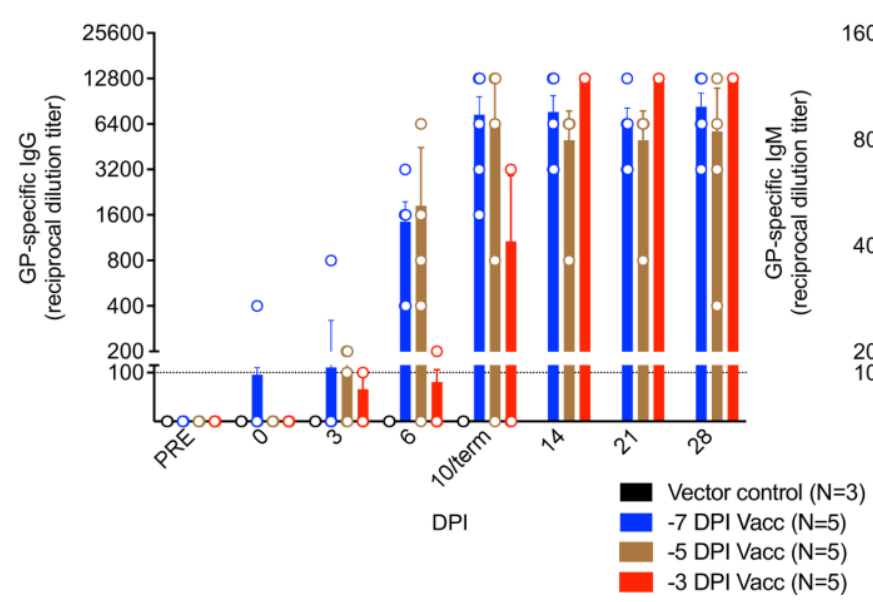

B

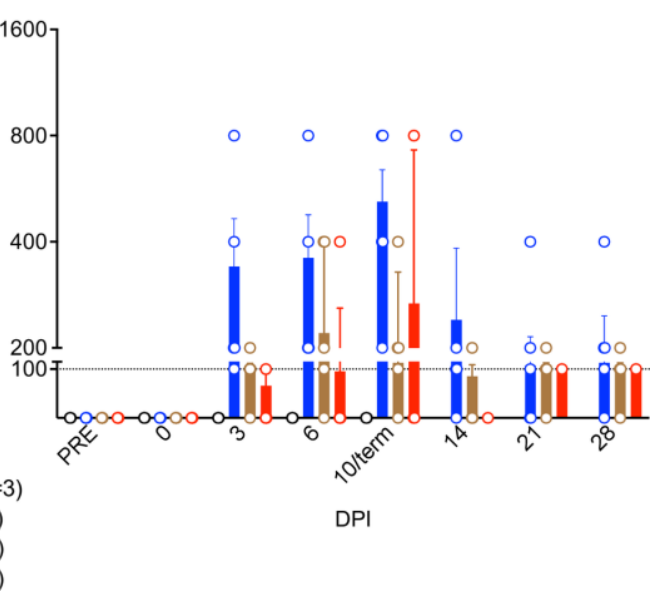

543 Fig 6. Reciprocal endpoint dilution titers of anti-MARV GP IgG and IgM in

\section{Vesiculovax-vaccinated cynomolgus macaques.}

545 MARV GP-specific (A) IgG and (B) IgM titers in vaccinated cohorts immunized with

546 rVSV-N4CT1-MARV-GP at -7 DPI (blue; $\mathrm{n}=5$ ), -5 DPI (brown; $\mathrm{n}=5$ ), or -3DPI (red; $\mathrm{n=5}$ ),

547 or a vector control (black; $n=3$ ). The mean titer \pm SEM is shown for each group at the 
548 denoted time points. Abbreviations: IgG, immunoglobulin G; IgM, immunoglobulin M;

549 DPI, days post infection; term, terminal; MARV, Marburg virus; GP, glycoprotein; PRE,

550 pre-vaccination baseline.

551

\section{Discussion}

553 In a prior study, we showed that a highly attenuated rVSV-based vector, rVSV-N4CT1-

554 MARV-GP, administered 20-30 minutes postexposure to a low dose MARV-Angola

555 challenge afforded partial protection (60\% survival) of NHPs [14]. As an extension of this

556 previous work, we determined the minimum time needed between immunization and

557 exposure to fully protect NHPs against MVD. Here, vaccination of macaques with a single

55810 million PFU dose of rVSV-N4CT1-MARV-GP one week prior to MARV-Angola

559 challenge resulted in lack of detectable viremia in these animals with protection from

560 clinical disease and lethality. [27]. However, we detected changes in bloodwork of

561 surviving subjects indicative of very mild disease in the absence of clinical signs.

562 Immunization at 5 or 3 days before exposure resulted in $80 \%$ and $20 \%$ survival,

563 respectively. Thus, this highly attenuated Vesiculovax vector provided comparable

564 protection (100\% survival) as the delta $\mathrm{G}$ vector as a rapid vaccine when administered one

565 week before exposure. Although higher survival was reported for the delta G vector when

566 given -3 days pre-challenge; no statistically significant difference was found between the

567 two vaccines (log rank test, data not shown). Small group sizes used in the previous study

568 make a direct comparison of vaccine efficacy difficult to interpret. Nevertheless, the fast-

569 acting protection of rVSV-N4CT1-MARV-GP supports the use of Vesiculovax vaccines

570 to combat future outbreaks of MVD. As a testament to this potential, ring vaccination with 
571 a similar EBOV-specific rVSV vector, Ervebo, reduced mortality and virus transmission

572 during the 2013-2016 West Africa EBOV epidemic and 2018-2020 DRC EBOV outbreak

$573[11,12]$.

574 While the Ervebo "delta G (rVSV $\Delta \mathrm{G})$ " vaccine appears highly efficacious, some

575 undesirable vaccine-associated events have been reported in humans, particularly in

576 individuals receiving high doses. Besides common vaccine reactions (injection site pain,

577 fatigue, headache, muscle pain), these side effects included moderate to severe arthritis

578 (with pain lasting a median of 8 days), postvaccination fever persisting for days, cutaneous

579 vasculitis, and dermatitis manifesting as vesicular, maculopapular, or purpuric lesions

580 distal to the inoculation site [28, 29]. Recovery of virus from synovial fluid and skin

581 vesicles confirmed dissemination of the vaccine vector in peripheral tissues. Lowering the

582 dose of Ervebo reduced reactogenicity, but also decreased immunogenicity and did not

583 prevent arthritis, dermatitis, or cutaneous vasculitis in vaccinees. Although replication of

584 the vaccine vector likely contributes to the rapid protection achieved with Ervebo, high

585 viral loads throughout the body raises concerns about the potential short- and long-term

586 effects of the vaccine. For these reasons, Ervebo is primarily indicated for reactive

587 vaccination of individuals at high risk of exposure. A vaccine with comparably efficacy

588 but a safer profile would be preferred for widespread use. Indeed, a rVSV-N4CT1 vector

589 expressing EBOV GP protein similarly protected NHPs from lethal EBOV challenge

590 despite a high degree of attenuation [41,42] and was well tolerated and immunogenic in

591 phase I clinical evaluation (https://clinicaltrials.gov/ct2/show/NCT02718469) [30]. The

592 vaccine elicited robust EBOV GP-specific IgG responses and modest but balanced cellular

593 immune responses. Importantly, no incidents of vaccine shedding, arthritis, nor skin rashes 
594 were reported in vaccinees at any dose tested including individuals given a high 18 million

595 PFU dose. Based on these results, we anticipate rVSV-N4CT1-MARV-GP can similarly

596 serve as a safe, immunogenic, and effective vaccine against MVD suitable for both

597 preventive and reactive immunization.

598 The specific mechanisms of rapid rVSV-elicited protection are not fully elucidated

599 but are thought to involve stimulation of both innate and adaptive immunity [13, 27, 43-

600 45]. Resistance to disease was strongly tied to early control of MARV replication. On the

601 day of challenge, specifically vaccinated survivors compared to fatal cases tended to

602 express higher levels of transcripts associated with interferon and RIG-I signaling,

603 indicating a robust innate response may be needed to shape protective immunity. In

604 survivors, innate signaling promptly resolved after MARV exposure unlike fatal cases

605 concurrently with an accumulation of adaptive immunity-related transcripts, particularly

606 in earlier vaccinated subjects. Administration of a non-specific rVSVN4CT1-HIV-gag1

607 vector control did not appear to delay the time-to-death and resulted in complete lethality

608 of macaques following exposure to MARV-Angola, indicating MARV GP-specific

609 responses modulate host resistance to MVD. This hypothesis is supported by the lack of

610 substantial antibody titers and the absence of adaptive signaling in vector control subjects.

611 Conversely, MARV GP-specific IgM and IgG titers were detected in all specifically

612 vaccinated survivors by 0-6 DPI, and increased transcriptionally derived B cell, cytotoxic

613 cell, and Th1 cell predicted frequencies were detected in these subjects as early as the day

614 of challenge. Thus, humoral and cellular immune responses directed at the GP correlate

615 with survival as we have previously shown [13]. 
616 Fatal outcome in vaccinated subjects corresponded with dramatic upregulation of

617 transcripts encoding pro-inflammatory IP-10 and neutrophil-associated S100A8 and

618 S100A9, which were previous reported to correlate with EBOV lethality in humans [36,

619 37] and in NHPs infected with Bundibugyo ebolavirus [33]. Similarly, lethality correlated

620 with expression of immune checkpoint molecules as reported in other studies (LAG3 [13],

621 CTLA4 [38]). Thus, certain factors associated with host susceptibility to MVD appear

622 shared among various filovirus infections. The most significantly downregulated mRNA

623 in vector control and vaccinated non-surviving subjects was complement factor $\mathrm{D}(C F D)$,

624 a serine protease that cleaves complement factor B in the alternative complement pathway

625 leading to downstream activation of the membrane attack complex [46]. As CFD is known

626 to participate in opsonization and killing of pathogens, and viruses are commonly known

627 to evade the complement system, this novel filovirus disease mechanism should be further

628 explored.

629 Our transcriptomic results revealed NK cells and cytotoxic T cells are strongly

630 implicated in rVSV-N4CT1-MARV-GP-associated fast-acting protection. Transcripts

631 encoding cytoplasmic granules including perforin (PRF1), granzyme A (GZMA),

632 granzyme B $(G Z M A)$, granzyme $\mathrm{K}(G Z M A)$, and granzyme $\mathrm{H}(G Z M H)$ were among the

633 topmost upregulated molecules in vaccinated survivors. Not until late disease did we

634 observe granzyme expression in fatal subjects. Activation of lymphocytes in non-survivors

635 likely represents cytokine-mediated and not antigen-dependent T-cell activation as

636 filovirus infection is known to interfere with antigen presentation [47, 48], and these

637 subjects exhibited high viral loads. Increased expression of transcripts encoding killer cell

638 lectin-type receptors (KLRF1 (NKp80), KLRB1 (CD161), KLRD1 (CD94)) was also 
639 evident in surviving subjects. These receptors are ubiquitously expressed on NK cells and

640 are involved in stimulation and regulation of cytotoxicity or self-nonself discrimination,

641 further supporting this claim.

642 Previously, we showed higher overall NK cell frequencies and recruitment of a

643 specific subset $\left(\mathrm{CD}^{+} 6^{+}\right)$of NK cells were important for postexposure protection against

644 MVD with rVSV $\Delta \mathrm{G}$ and rVSV-N2CT1 vectors [13]. CD16 ${ }^{+}$CD8 $\mathrm{a}^{+} \mathrm{NK}$ cells in NHPs

645 represent the equivalent of the highly cytotoxic CD56 $6^{\mathrm{dim}}$ NK cell subset in humans [40].

646 These cells have limited cytokine-secreting potential but participate in killing virally

647 infected cells or initiating antibody-dependent cell-mediated cytotoxicity (ADCC).

648 Accordingly, depletion studies have revealed NK cell-intact mice survive longer after a

649 mouse-adapted EBOV challenge, which is further enhanced by postexposure treatment

650 with a rVSV $\Delta$ G expressing EBOV GP (VSV $\Delta$ G/EBOV GP) [49]. The authors of this study

651 reported treatment with VSV $\Delta$ G/EBOV GP treatment resulted in significantly higher NK

652 cell-mediated cytotoxicity and IFN- $\gamma$ secretion. Furthermore, recent research using a

653 systems biology approach has identified NK cells as a primary correlate of antibody

654 induction in humans receiving Ervebo. [44]. Specifically, the authors found that the

655 frequency of CD56 $6^{\text {bright }} \mathrm{NK}$ cells on day 3 postvaccination and the expression of CXCR6

656 on $\mathrm{CD}^{\mathrm{dim}} \mathrm{NK}$ cells on day 1 postvaccination positively correlated with antibody

657 responses suggesting a potential role for antibody-mediated NK cell activation in vaccine-

658 induced immune responses [50]. Thus, early NK cell differentiation status may dictate Fc-

659 mediated activation of NK cells. This finding may be of relevance as antibody neutralizing

660 capacity is not highly correlative of protection against MVD, and only low levels of

661 neutralizing antibodies are typically detected following rVSV-MARV vaccination [13, 27]. 
662 Antibody neutralizing capacity and non-neutralizing antibody mechanisms were not

663 assessed in this study, but future examinations should examine the role of ADCC and other

664 Fc-mediated antibody effector functions in rVSV-N4CT1-MARV-GP-mediated protection

665 against MVD.

666 Other immune components may also confer rVSV-N4CT1-MARV-GP protection.

667 Results from clinical trials showed VSV $\triangle \mathrm{G} / \mathrm{EBOV}$ GP elicited GP-specific CTLs, 668 follicular T helper cells, and IFN- $\gamma$-secreting T helper cells [51-53]. Not surprisingly, Tbet

669 (TBX21), the lineage-defining transcription factor for Th1 cells [54], was one of the most

670 significantly upregulated transcripts in survivors 3-10 DPI in this study. Th1 cells secrete

671 IFN- $\gamma$ and IL-2 and represent a lineage of $\mathrm{CD}^{+}$effector T cells that promote cell-mediated

672 immunity and defense against intracellular pathogens. As we've previously reported,

673 postexposure survival against MVD following rVSV vaccination corresponded with

674 increased polyfunctional IFN- $\gamma^{+}$and IL-2 ${ }^{+}$MARV GP-specific Th1 cells [13]. Other

675 effector functions of Tbet include promotion of 1) immunoglobulin class switching, 2) the

676 terminal differentiation of CD8+ T cells, 3) the maturation of NK cells, and 4) the secretion

677 of cytoplasmic granules such as granzymes (46). Additionally, Tbet can protect the host

678 from amplification of aberrant innate responses by dampening type-I IFN transcription

679 factors and IFN-stimulated genes [55], which are highly expressed after MARV-Angola

680 infection [56] and corresponded with lethal outcome in this study. Our pathway analysis

681 supported activation of T cell responses and simultaneous repression of pathways involved

682 in pattern recognition receptor and interferon signaling in line with this reasoning. Based

683 on these collective findings, we infer virus-specific cytotoxic and helper T cells, along with

684 plasma cells and NK cells, likely play a key role in defense against MVD. 
686 additional clarification, we demonstrate that innate and adaptive immunity likely act in

687 concert to elicit fast-acting protection. Further investigation is needed to determine how

688 individual host responses to rVSV-N4CT1-MARV-GP vaccination alter susceptibility to

689 MVD. In conclusion, the rapid protection mediated by rVSV-N4CT1-MARV-GP, along

690 with promising safety and immunogenicity data from a phase I clinical evaluation with an

691 analogous rVSV-N4CT1 vaccine, supports the testing of rVSV-N4CT1-MARV-GP in

692 phase I clinical trials as the next step in developing an effective and safe vaccine against

693 MARV. Our results indicate highly attenuated Vesiculovax vaccines may be suitable for

694 ring vaccination during MVD outbreaks to save lives and reduce community transmission.

695

\section{Acknowledgements}

697 The authors wish to thank the UTMB Animal Resource Center for husbandry support of

698 laboratory animals. We wish to thank Drs. Kevin Melody and Abhishek Prasad for

699 assistance with the animal experiments. 


\section{References}

701

702 1. Woolsey C, Geisbert, TW, and Cross, RW. Marburg and Ravn Viruses

703 (Filoviridae). Bamford D, Zuckerman, M, editor. Encylopedia of Virology, 4th edition:

704 Oxford: Academic Press Vol 2 (608-618); 2021.

705 2. WHO. 2018 Annual review of diseases prioritized under the Research and

706 Development Blueprint. Geneva, Switzerland. Accessed 09 Dec 2021:

707 https://www.who.int/emergencies/diseases/2018prioritization-report.pdf. 2018.

708 3. CDC/USDA. Federal Select Agent Program: HHS and USDA Select Agents and 709 Toxins 7CFR Part 331, 9 CFR Part 121, and 42 CFR Part 73. Accessed 09 Dec 2021: 710 https://www.selectagents.gov/sat/list.htm?CDC_AA_refVal=https\%3A\%2F\%2Fwww.sel 711 ectagents.gov\%2FSelectAgentsandToxinsList.html. 2021.

712 4. Towner JS, Khristova ML, Sealy TK, Vincent MJ, Erickson BR, Bawiec DA, et al. 713 Marburgvirus Genomics and Association with a Large Hemorrhagic Fever Outbreak in 714 Angola. Journal of Virology. 2006;80(13):6497-516. doi: 10.1128/jvi.00069-06.

715 5. Towner JS, Pourrut X, Albariño CG, Nkogue CN, Bird BH, Grard G, et al. Marburg 716 Virus Infection Detected in a Common African Bat. PLoS ONE. 2007;2(8):e764. doi: 717 10.1371/journal.pone.0000764.

718 6. Amman BR, Carroll SA, Reed ZD, Sealy TK, Balinandi S, Swanepoel R, et al. 719 Seasonal Pulses of Marburg Virus Circulation in Juvenile Rousettus aegyptiacus Bats 720 Coincide with Periods of Increased Risk of Human Infection. PLoS Pathogens. 721 2012;8(10):e1002877. doi: 10.1371/journal.ppat.1002877.

722 7. WHO. Press Release 09 Aug 2021: West Africa's first-ever case of Marburg virus

723 disease confirmed in Guinea. Accessed 09 Dec 2021:

724 https://worldhealthorganization.cmail19.com/t/ViewEmail/d/85906C6ABA3E08E32540

725 EF23F30FEDED/84D956050C9556035281BC0AA5ABFD98. 2021. Epub 09 Aug 2021.

726 8. Amman BR, Bird BH, Bakarr IA, Bangura J, Schuh AJ, Johnny J, et al. Isolation 727 of Angola-like Marburg virus from Egyptian rousette bats from West Africa. Nature 728 Communications. 2020;11(1). doi: 10.1038/s41467-020-14327-8.

729 9. Woolsey C, Geisbert TW. Current state of Ebola virus vaccines: A snapshot. PLOS 730 Pathogens. 2021;17(12):e1010078. doi: 10.1371/journal.ppat.1010078.

731 10. Choi MJ, Cossaboom CM, Whitesell AN, Dyal JW, Joyce A, Morgan RL, et al. 732 Use of Ebola Vaccine: Recommendations of the Advisory Committee on Immunization 733 Practices, United States, 2020. MMWR Recommendations and Reports. 2021;70(1):1-12. 734 doi: 10.15585/mmwr.rr7001a1. 
735

736

737

738

739

740

741

742

743

744

745

746

747

748

749

750

751

752

753

754

755

756

757

758

759

760

761

762

763

764

765

766

767

768

769

770

771

772 20. Daddario-Dicaprio KM, Geisbert TW, Geisbert JB, StröHer U, Hensley LE, Grolla 773 A, et al. Cross-Protection against Marburg Virus Strains by Using a Live, Attenuated

11. WHO. Preliminary results on the efficacy of rVSV-ZEBOV-GP Ebola vaccine using the ring vaccination strategy in the control of an Ebola outbreak in the Democratic Republic of the Congo: an example of integration of research into epidemic response. 10 April 2019 meeting report. Accessed 04 Nov 21. Available from: https://www.who.int/csr/resources/publications/ebola/ebola-ring-vaccination-results-12april-2019.pdf.

12. Henao-Restrepo AM, Camacho A, Longini IM, Watson CH, Edmunds WJ, Egger $\mathrm{M}$, et al. Efficacy and effectiveness of an rVSV-vectored vaccine in preventing Ebola virus disease: final results from the Guinea ring vaccination, open-label, cluster-randomised trial (Ebola Ça Suffit!). The Lancet. 2017;389(10068):505-18. doi: 10.1016/s01406736(16)32621-6.

13. Woolsey C, Jankeel A, Matassov D, Geisbert JB, Agans KN, Borisevich V, et al. Immune correlates of postexposure vaccine protection against Marburg virus. Scientific Reports. 2020;10(1). doi: 10.1038/s41598-020-59976-3.

14. Woolsey C, Geisbert JB, Matassov D, Agans KN, Borisevich V, Cross RW, et al. Postexposure Efficacy of Recombinant Vesicular Stomatitis Virus Vectors Against High and Low Doses of Marburg Virus Variant Angola in Nonhuman Primates. The Journal of Infectious Diseases. 2018;218(suppl_5):S582-S7. doi: 10.1093/infdis/jiy293.

15. Geisbert TW, Hensley LE, Geisbert JB, Leung A, Johnson JC, Grolla A, et al. Postexposure Treatment of Marburg Virus Infection. Emerging Infectious Diseases. 2010;16(7):1119-22. doi: 10.3201/eid1607.100159.

16. Geisbert TW, Feldmann H. Recombinant Vesicular Stomatitis Virus-Based Vaccines Against Ebola and Marburg Virus Infections. The Journal of Infectious Diseases. 2011;204(suppl_3):S1075-S81. doi: 10.1093/infdis/jir349.

17. Matassov D, Mire, C. E., Latham, T., Geisbert, J. B., Xu, R., Ota-Setlik, A., Agans, K. N., Kobs, D. J., Wendling, M., Burnaugh, A., Rudge, T. L., Jr, Sabourin, C. L., Egan, M. A., Clarke, D. K., Geisbert, T. W., \& Eldridge, J. H. . Single-Dose Trivalent VesiculoVax Vaccine Protects Macaques from Lethal Ebolavirus and Marburgvirus Challenge. Journal of virology. 2018;92(3), e01190-17.

18. Geisbert TW, Geisbert, J. B., Leung, A., Daddario-DiCaprio, K. M., Hensley, L. E., Grolla, A., \& Feldmann, H. Single-injection vaccine protects nonhuman primates against infection with marburg virus and three species of ebola virus. Journal of virology. 2009;83(14), 7296-7304.

19. Mire CE, Geisbert JB, Versteeg KM, Mamaeva N, Agans KN, Geisbert TW, et al. A Single-Vector, Single-Injection Trivalent Filovirus Vaccine: Proof of Concept Study in Outbred Guinea Pigs. Journal of Infectious Diseases. 2015;212(suppl 2):S384-S8. doi: 10.1093/infdis/jiv126. 
774 Recombinant Vaccine. Journal of Virology. 2006;80(19):9659-66. doi: 10.1128/jvi.00959-

77506.

776 21. Daddario-Dicaprio KM, Geisbert TW, Ströher U, Geisbert JB, Grolla A, Fritz EA, 777 et al. Postexposure protection against Marburg haemorrhagic fever with recombinant 778 vesicular stomatitis virus vectors in non-human primates: an efficacy assessment. The Lancet. 2006;367(9520):1399-404. doi: 10.1016/s0140-6736(06)68546-2.

780 22. Geisbert TW, Strong JE, Feldmann H. Considerations in the Use of Nonhuman 781 Primate Models of Ebola Virus and Marburg Virus Infection: Table 1. Journal of Infectious 782 Diseases. 2015;212(suppl 2):S91-S7. doi: 10.1093/infdis/jiv284.

783 23. Mire CE, Geisbert JB, Agans KN, Satterfield BA, Versteeg KM, Fritz EA, et al. 784 Durability of a Vesicular Stomatitis Virus-Based Marburg Virus Vaccine in Nonhuman 785 Primates. PLoS ONE. 2014;9(4):e94355. doi: 10.1371/journal.pone.0094355.

786 24. Matsuno K, Kishida N, Usami K, Igarashi M, Yoshida R, Nakayama E, et al. 787 Different Potential of C-Type Lectin-Mediated Entry between Marburg Virus Strains. 788 Journal of Virology. 2010;84(10):5140-7. doi: 10.1128/jvi.02021-09.

789 25. Cross RW, Fenton KA, Geisbert JB, Ebihara H, Mire CE, Geisbert TW. 790 Comparison of the Pathogenesis of the Angola and Ravn Strains of Marburg Virus in the 791 Outbred Guinea Pig Model. Journal of Infectious Diseases. 2015;212(suppl 2):S258-S70. 792 doi: 10.1093/infdis/jiv182. with recombinant nematode anticoagulant protein c2. J Infect Dis. 2007;196 Suppl 2(Suppl 2):S372-81. doi: 10.1086/520608. PubMed PMID: 17940973; PubMed Central PMCID: PMCPMC7110112.

27. Marzi A JA, Menicucci AR, Callison J, O’Donnell KL, Feldmann F, Pinski AN, Hanley PW, Messaoudi I. Single Dose of a VSV-Based Vaccine Rapidly Protects Macaques From Marburg Virus Disease. Frontiers in immunology. 2021;12.

801 28. Huttner A, Dayer J-A, Yerly S, Combescure C, Auderset F, Desmeules J, et al. The 802 effect of dose on the safety and immunogenicity of the VSV Ebola candidate vaccine: a 803 randomised double-blind, placebo-controlled phase 1/2 trial. The Lancet Infectious 804 Diseases. 2015;15(10):1156-66. doi: 10.1016/s1473-3099(15)00154-1.

805 29. Agnandji ST, Huttner A, Zinser ME, Njuguna P, Dahlke C, Fernandes JF, et al. 806 Phase 1 Trials of rVSV Ebola Vaccine in Africa and Europe. New England Journal of 807 Medicine. 2016;374(17):1647-60. doi: 10.1056/nejmoa1502924.

808 30. Clarke DK, Xu R, Matassov D, Latham TE, Ota-Setlik A, Gerardi CS, et al. Safety 809 and immunogenicity of a highly attenuated rVSVN4CT1-EBOVGP1 Ebola virus vaccine: 810 a randomised, double-blind, placebo-controlled, phase 1 clinical trial. The Lancet 811 Infectious Diseases. 2020;20(4):455-66. doi: 10.1016/s1473-3099(19)30614-0. 
812 31. Wertz GW, Perepelitsa VP, Ball LA. Gene rearrangement attenuates expression 813 and lethality of a nonsegmented negative strand RNA virus. Proceedings of the National 814 Academy of Sciences. 1998;95(7):3501-6. doi: 10.1073/pnas.95.7.3501.

815 32. Roberts A, Buonocore L, Price R, Forman J, Rose JK. Attenuated vesicular 816 stomatitis viruses as vaccine vectors. Journal of virology. 1999;73(5):3723-32. doi: 817 10.1128/JVI.73.5.3723-3732.1999. PubMed PMID: 10196265.

818 33. Woolsey C, Borisevich V, Agans KN, Fenton KA, Cross RW, Geisbert TW. 819 Bundibugyo ebolavirus Survival Is Associated with Early Activation of Adaptive 820 Immunity and Reduced Myeloid-Derived Suppressor Cell Signaling. mBio. 821 2021;12(4):e0151721. Epub 20210810. doi: 10.1128/mBio.01517-21. PubMed PMID: 822 34372693; PubMed Central PMCID: PMCPMC8406165.

823 34. Stelzer G, Rosen N, Plaschkes I, Zimmerman S, Twik M, Fishilevich S, et al. The 824 GeneCards Suite: From Gene Data Mining to Disease Genome Sequence Analyses. 825 Current Protocols in Bioinformatics. 2016;54(1):1.30.1-1..3. doi: 826 https://doi.org/10.1002/cpbi.5.

827 35. Geisbert TW, Bailey M, Geisbert JB, Asiedu C, Roederer M, Grazia-Pau M, et al. 828 Vector choice determines immunogenicity and potency of genetic vaccines against Angola 829 Marburg virus in nonhuman primates. J Virol. 2010;84(19):10386-94. Epub 20100721. 830 doi: 10.1128/jvi.00594-10. PubMed PMID: 20660192; PubMed Central PMCID: 831 PMCPMC2937810.

832 36. McElroy AK, Erickson BR, Flietstra TD, Rollin PE, Nichol ST, Towner JS, et al. 833 Ebola Hemorrhagic Fever: Novel Biomarker Correlates of Clinical Outcome. Journal of 834 Infectious Diseases. 2014;210(4):558-66. doi: 10.1093/infdis/jiu088.

835 37. Wauquier N, Becquart P, Padilla C, Baize S, Leroy EM. Human Fatal Zaire Ebola 836 Virus Infection Is Associated with an Aberrant Innate Immunity and with Massive 837 Lymphocyte Apoptosis. PLoS Neglected Tropical Diseases. 2010;4(10):e837. doi: 838 10.1371/journal.pntd.0000837.

839 38. Ruibal P, Oestereich L, Lüdtke A, Becker-Ziaja B, Wozniak DM, Kerber R, et al. 840 Unique human immune signature of Ebola virus disease in Guinea. Nature. 841 2016;533(7601):100-4. doi: 10.1038/nature17949.

842 39. Ries M, Reynolds MR, Bashkueva K, Crosno K, Capuano S, III, Prall TM, et al. 843 KIR3DL01 upregulation on gut natural killer cells in response to SIV infection of KIR844 and MHC class I-defined rhesus macaques. PLOS Pathogens. 2017;13(7):e1006506. doi: 845 10.1371/journal.ppat.1006506.

846 40. Webster RL, Johnson RP. Delineation of multiple subpopulations of natural killer 847 cells in rhesus macaques. Immunology. 2005;115(2):206-14. doi: 10.1111/j.1365848 2567.2005.02147.x. 
849 41. Mire CE, Matassov D, Geisbert JB, Latham TE, Agans KN, Xu R, et al. Single850 dose attenuated Vesiculovax vaccines protect primates against Ebola Makona virus. 851 Nature. 2015;520(7549):688-91. doi: 10.1038/nature14428.

852 42. Matassov D, Marzi A, Latham T, Xu R, Ota-Setlik A, Feldmann F, et al. 853 Vaccination With a Highly Attenuated Recombinant Vesicular Stomatitis Virus Vector 854 Protects Against Challenge With a Lethal Dose of Ebola Virus. Journal of Infectious 855 Diseases. 2015;212(suppl 2):S443-S51. doi: 10.1093/infdis/jiv316.

856 43. Pejoski D, De Rham C, Martinez-Murillo P, Santoro F, Auderset F, Medaglini D, 857 et al. Rapid dose-dependent Natural Killer (NK) cell modulation and cytokine responses 858 following human rVSV-ZEBOV Ebolavirus vaccination. npj Vaccines. 2020;5(1). doi: 859 10.1038/s41541-020-0179-4.

860 44. Rechtien A, Richert L, Lorenzo H, Martrus G, Hejblum B, Dahlke C, et al. Systems 861 Vaccinology Identifies an Early Innate Immune Signature as a Correlate of Antibody 862 Responses to the Ebola Vaccine rVSV-ZEBOV. Cell Reports. 2017;20(9):2251-61. doi: 863 https://doi.org/10.1016/j.celrep.2017.08.023.

864 45. Medaglini D, Santoro F, Siegrist C-A. Correlates of vaccine-induced protective 865 immunity against Ebola virus disease. Seminars in Immunology. 2018;39:65-72. doi: 866 https://doi.org/10.1016/j.smim.2018.07.003.

867 46. Stoermer KA, Morrison TE. Complement and viral pathogenesis. Virology. 868 2011;411(2):362-73. Epub 2011/02/02. doi: 10.1016/j.virol.2010.12.045. PubMed PMID: 86921292294.

870 47. Kotliar D, Lin AE, Logue J, Hughes TK, Khoury NM, Raju SS, et al. Single-Cell 871 Profiling of Ebola Virus Disease In Vivo Reveals Viral and Host Dynamics. Cell. 872 2020;183(5):1383-401.e19. doi: 10.1016/j.cell.2020.10.002.

873 48. Mohamadzadeh M, Chen L, Schmaljohn AL. How Ebola and Marburg viruses 874 battle the immune system. Nature Reviews Immunology. 2007;7(7):556-67. doi: $87510.1038 /$ nri2098.

876 49. Williams KJ, Qiu X, Fernando L, Jones SM, Alimonti JB. VSV $\Delta$ G/EBOV GP877 induced innate protection enhances natural killer cell activity to increase survival in a lethal 878 mouse adapted Ebola virus infection. Viral immunology. 2015;28(1):51-61.

879 50. Wagstaffe HR, Clutterbuck EA, Bockstal V, Stoop JN, Luhn K, Douoguih M, et al. 880 Antibody-Dependent Natural Killer Cell Activation After Ebola Vaccination. The Journal 881 of Infectious Diseases. 2021;223(7):1171-82. doi: 10.1093/infdis/jiz657.

882 51. Dahlke C, Kasonta R, Lunemann S, Krähling V, Zinser ME, Biedenkopf N, et al. 883 Dose-dependent T-cell Dynamics and Cytokine Cascade Following rVSV-ZEBOV 884 Immunization. EBioMedicine. 2017;19:107-18. doi: 10.1016/j.ebiom.2017.03.045. 
885 52. Farooq F, Beck K, Paolino KM, Phillips R, Waters NC, Regules JA, et al.

886 Circulating follicular $\mathrm{T}$ helper cells and cytokine profile in humans following vaccination

887 with the rVSV-ZEBOV Ebola vaccine. Scientific Reports. 2016;6(1):27944. doi:

$88810.1038 /$ srep27944.

889 53. Medaglini D, Santoro F, Siegrist CA. Correlates of vaccine-induced protective 890 immunity against Ebola virus disease. Semin Immunol. 2018;39:65-72. Epub 20180721. 891 doi: 10.1016/j.smim.2018.07.003. PubMed PMID: 30041831.

892 54. Lazarevic V, Glimcher LH, Lord GM. T-bet: a bridge between innate and adaptive 893 immunity. Nature Reviews Immunology. 2013;13(11):777-89. doi: 10.1038/nri3536.

894 55. Iwata S, Mikami Y, Sun H-W, Brooks SR, Jankovic D, Hirahara K, et al. The 895 Transcription Factor T-bet Limits Amplification of Type I IFN Transcriptome and 896 Circuitry in $\mathrm{T}$ Helper 1 Cells. Immunity. 2017;46(6):983-91.e4. doi: 897 10.1016/j.immuni.2017.05.005.

898 56. Connor JH, Yen J, Caballero IS, Garamszegi S, Malhotra S, Lin K, et al. 899 Transcriptional Profiling of the Immune Response to Marburg Virus Infection. J Virol. 900 2015;89(19):9865-74. Epub 20150722. doi: 10.1128/jvi.01142-15. PubMed PMID: 901 26202234; PubMed Central PMCID: PMCPMC4577901. 


\section{Supporting Information Captions}

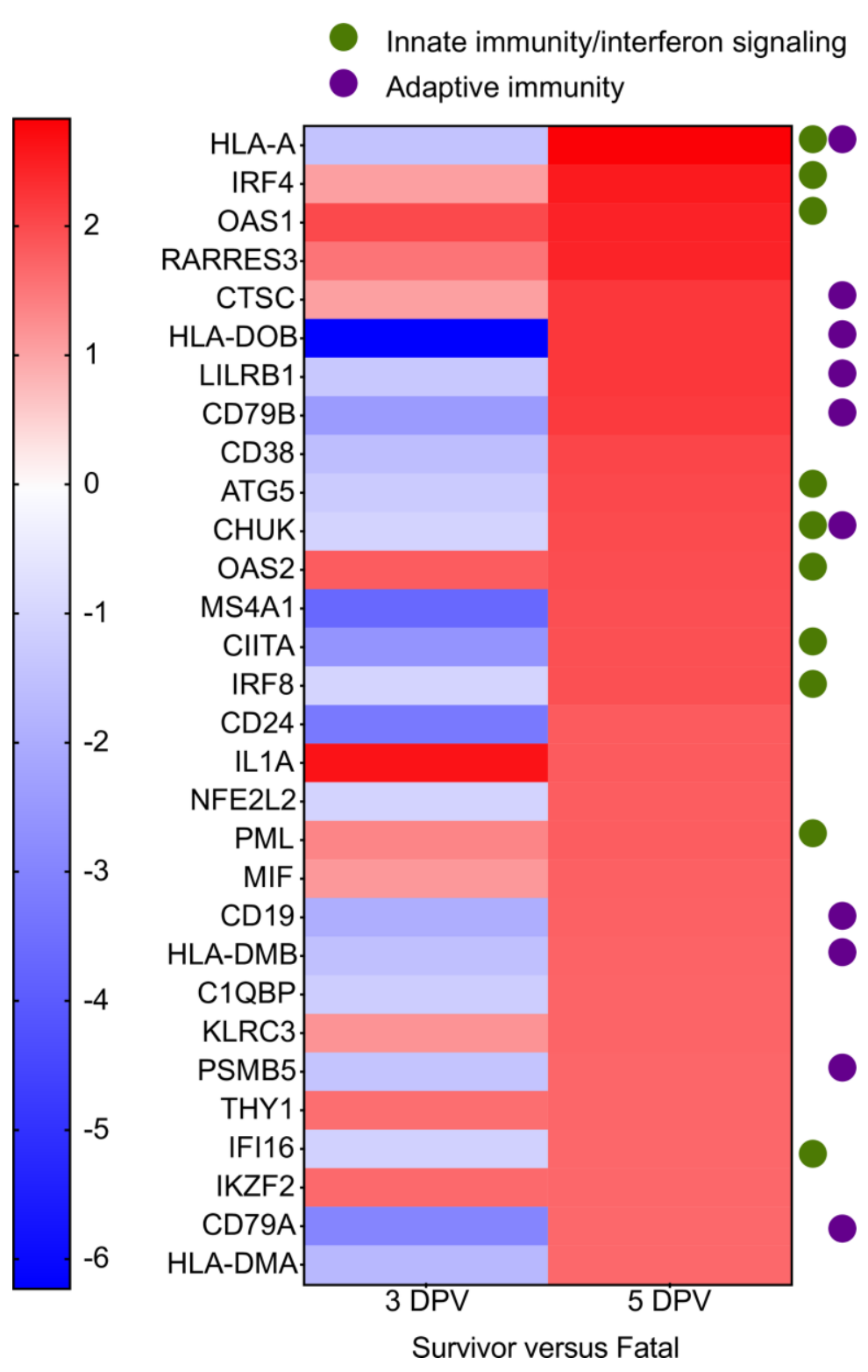

905 Fig S1. The topmost upregulated transcripts in Vesiculovax-vaccinated survivors on

906 the day of challenge. Heatmap of the most upregulated mRNAs in survivor versus fatal

907 subjects at five days post rVSV-N4CT1-MARV-GP vaccination (DPV) compared to 3

908 DPV. As only a single survivor exists in the 3 DPV group and a single fatal case exists in

909 the 5 DPV group, statistical analysis was not performed. Red indicates high expression;

910 blue indicates low expression; white indicates no difference in expression. Dots indicate 
911 whether specific mRNA is involved in adaptive immunity or innate immunity/interferon

912 signaling.

A

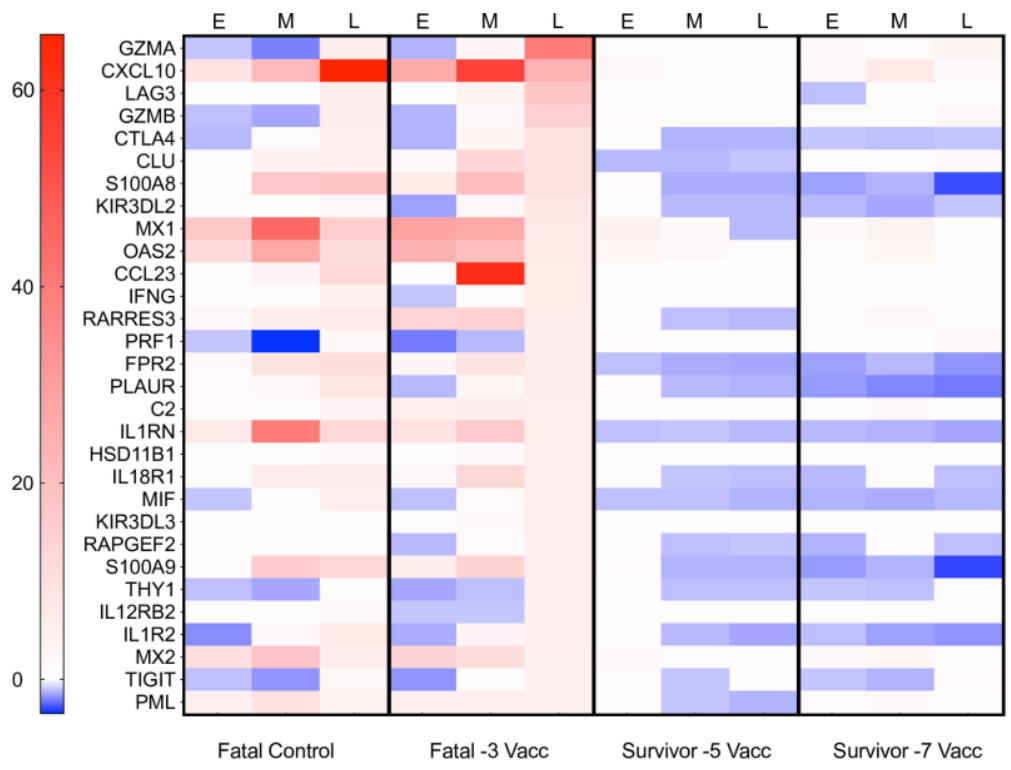

B

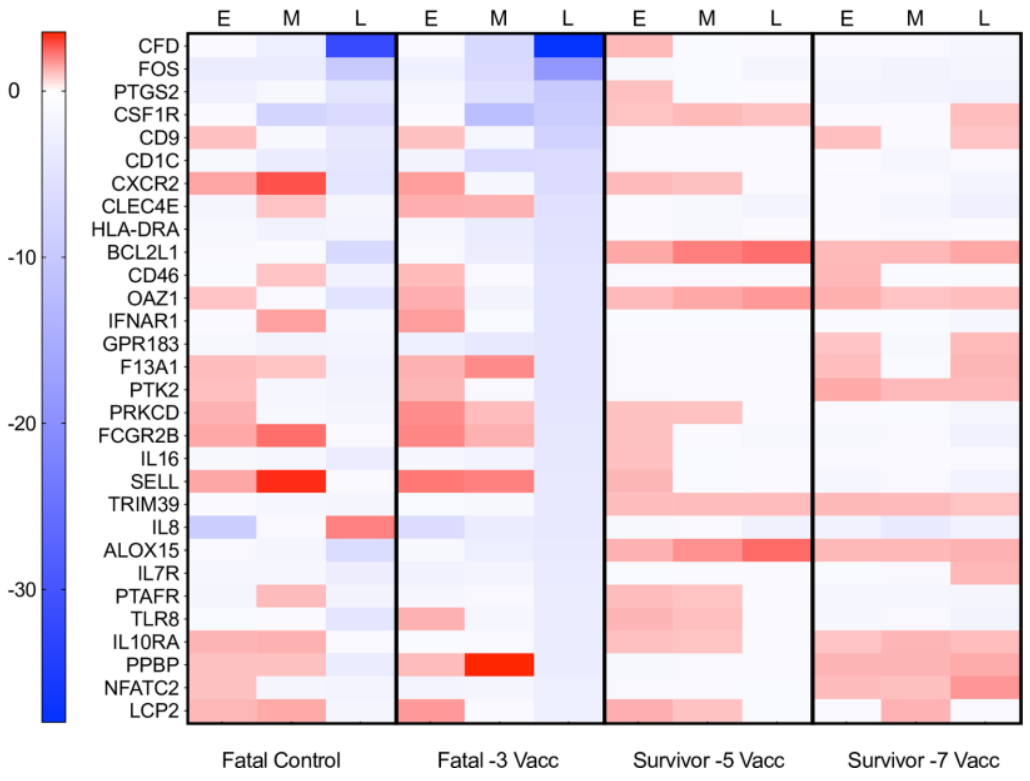

914 Fig S2. The topmost upregulated and downregulated transcripts in fatal subjects

\section{5 vaccinated with Vesiculovax vaccines.}

916 Heatmap of the most upregulated mRNAs in fatal subjects at five days post rVSV-N4CT1-

917 MARV-GP vaccination (DPV) compared to 3 DPV. As only a single survivor exists in the 
9183 DPV group and a single fatal case exists in the 5 DPV group, statistical analysis was not

919 performed. Red indicates high expression; blue indicates low expression; white indicates

920 no difference in expression. Dots indicate whether specific mRNA is involved in adaptive

921 immunity or innate immunity/interferon signaling. Abbreviations: Vacc, vaccinated; E,

922 early disease (3 DPI); M, mid disease (6 DPI); L, late disease (10 DPI or terminal

923 timepoint).

924

925

926
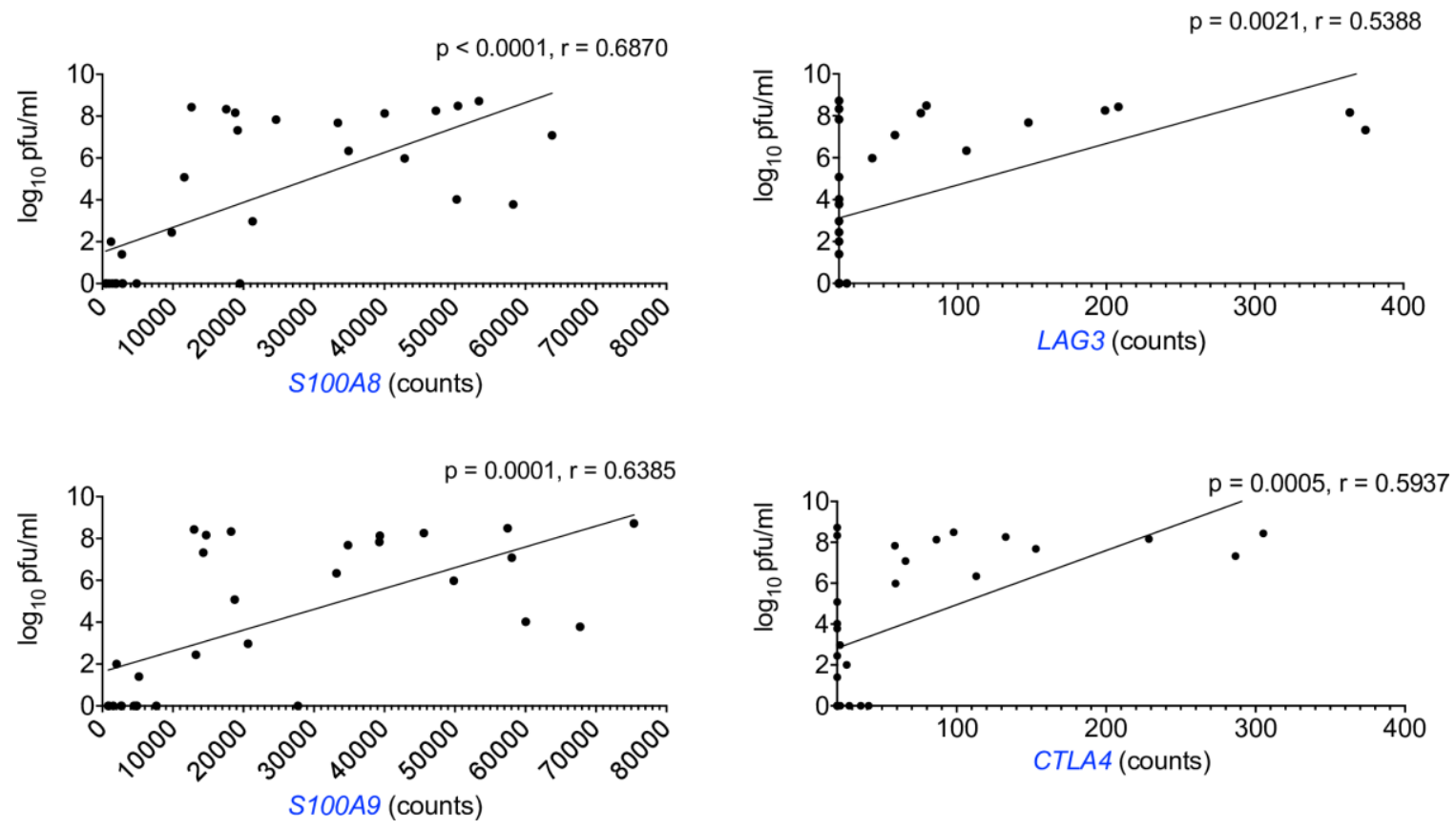

Fig S3. Transcriptional correlates associated with lethal outcome in Vesiculovax-

929 vaccinated macaques exposed to MARV-Angola.

930 Pearson correlation plots for calprotectin (S100A8 and S100A9) and immune checkpoint

931 (LAG3 and CTLA4) transcripts (Benjamini-Hochberg false discovery rate (FDR)

932 corrected p-value less than 0.05$)$. 


\begin{tabular}{|c|c|c|c|c|c|}
\hline $\begin{array}{l}\text { Animal ID } \\
\quad(\text { sex }) \\
\text { weight }\end{array}$ & $\begin{array}{c}\text { Group } \\
\text { (Day of } \\
\text { Vaccination) }\end{array}$ & $\begin{array}{l}\text { RT-qPCR } \\
\text { Titer }\left(\mathrm{LOG}_{10}\right. \\
\text { copies } / \mathrm{ml}^{*}\end{array}$ & $\begin{array}{c}\text { Viremia } \\
\text { Titer } \\
\left(\mathrm{LOG}_{10}\right. \\
\text { PFU/ml)* }\end{array}$ & Clinical Signs*† & $\begin{array}{l}\text { Final } \\
\text { Outcome }\end{array}$ \\
\hline $\begin{array}{l}\text { Control } 1 \\
181171 \\
\text { (M) } 2.86 \\
\quad \mathrm{~kg}\end{array}$ & -7 & $\begin{array}{l}7.25(3), 11.88 \\
(6), 11.71(8)\end{array}$ & $\begin{array}{l}5.08(3) \\
8.72(6) \\
8.43(8)\end{array}$ & $\begin{array}{c}\text { Anorexia (7,8), petechial rash (8), dyspnea }(8), \\
\text { lymphopenia (3), lymphocytosis }+(8), \text { monocytosis }+ \\
(6)+++(8), \text { neutropenia }(8), \text { neutrophilia }+(0,3,6), \\
\text { eosinopenia }(0,3) \text {, eosinophilia }+++(8), \text { basopenia }(3), \\
\text { basophilia }+(8), \text { BUN ++ }(8), \text { CRE }+++(8), \text { ALT }+++ \\
(6,8), \text { AST }+++(6,8), \text { ALP }++(6,8), \text { GGT }+++(6,8), \\
\text { CRP increase }(6,8)\end{array}$ & $\begin{array}{c}\text { Euthanized } 8 \\
\text { DPI }\end{array}$ \\
\hline $\begin{array}{l}\text { Survivor } 1 \\
181120 \\
\text { (M) } 3.36 \\
\mathrm{~kg}\end{array}$ & -7 & N.D. & N.D. & $\begin{array}{l}\text { Monocytopenia }(0,14) \text {, neutrophilia }++(0,14), \\
\text { eosinopenia }(21) \text {, basopenia }(21)\end{array}$ & Survived \\
\hline $\begin{array}{l}\text { Survivor } 2 \\
\text { Q1501008 } \\
\text { (F) } 3.88 \mathrm{~kg}\end{array}$ & -7 & N.D. & N.D. & Monocytopenia (14) & Survived \\
\hline $\begin{array}{l}\text { Survivor } 3 \\
142866 \\
\text { (M) } 7.60 \\
\mathrm{~kg}\end{array}$ & -7 & N.D. & N.D. & $\begin{array}{c}\text { Leukopenia }(0,6,10,14,21,28), \text { lymphocytosis }+(10), \\
\text { monocytopenia }(14,28), \text { neutropenia } \\
(0,3,6,10,14,21,28), \text { eosinopenia }(0,6,10,14,21), \\
\text { basopenia }(0,3,6,10,14,21,28)\end{array}$ & Survived \\
\hline $\begin{array}{c}\text { Survivor } 4 \\
131332(\mathrm{~F}) \\
3.94 \mathrm{~kg}\end{array}$ & -7 & N.D. & N.D. & $\begin{array}{c}\text { Anorexia (5), neutropenia (28), eosinopenia (6), } \\
\text { basopenia (6), ALT }+(0,3)++(10,21,28)+++(14), \\
\text { CRP increase }(6)\end{array}$ & Survived \\
\hline $\begin{array}{l}\text { Survivor } 5 \\
151022(\mathrm{~F}) \\
3.20 \mathrm{~kg} \\
\end{array}$ & -7 & N.D. & N.D. & $\begin{array}{c}\text { Neutropenia }(0,10) \text {, eosinopenia }(0,21) \text {, eosinophilia }+ \\
(28) \text {, basopenia }(0,21)\end{array}$ & Survived \\
\hline
\end{tabular}

933

934 S1 Table. Clinical findings in MARV-exposed cynomolgus macaques immunized with

\section{Vesiculovax vaccine 7 days prior to challenge.}

936 Macaques were immunized with a vector control (black; $n=1$ ) or rVSV-N4CT1-MARV-

937 GP vaccine at -7 DPI (blue; $n=5$ ). *Day after MARV challenge is in parentheses up to the

93828 DPI study endpoint. $†$ Fever is defined as a temperature greater than $2.5^{\circ} \mathrm{F}$ above

939 baseline, at least $1.5^{\circ} \mathrm{F}$ above baseline and $\geq 103.5^{\circ} \mathrm{F}$, or $1.1{ }^{\circ} \mathrm{F}$ above baseline and $\geq$

$940104^{\circ} \mathrm{F}$. Leukopenia, thrombocytopenia, and lymphopenia are defined by a $>40 \%$ drop in

941 numbers of leukocytes, platelets, and lymphocytes, respectively. Leukocytosis,

942 monocytosis, and granulocytosis are defined as a $\geq$ two-fold increase in leukocytes,

943 monocytes, and granulocytes, respectively. Crosses indicate increases in liver enzymes

944 (ALT, AST, ALP, GGT) or renal function test values (BUN, CRE): 2- to 3-fold increase, 
$945+$ +; >3- up to 5-fold increase, ++; >5-fold increase, +++. Abbreviations: M, male; F, female;

946 kg, kilogram; PFU, plaque-forming units; MARV, Marburg virus; BUN, blood urea

947 nitrogen; CRE, creatinine; ALT, alanine aminotransferase; AST, aspartate

948 aminotransferase; ALP, alkaline phosphatase; GGT, gamma-glutamyltransferase; CRP, c-

949 reactive protein; DPI, days post infection.

950

\begin{tabular}{|c|c|c|c|c|c|}
\hline $\begin{array}{l}\text { Animal ID } \\
\text { (sex) } \\
\text { weight }\end{array}$ & $\begin{array}{c}\text { Group } \\
\text { (Day of } \\
\text { Vaccination) }\end{array}$ & $\begin{array}{l}\text { RT-qPCR } \\
\text { Titer }\left(\mathrm{LOG}_{10}\right. \\
\text { copies/ml)* }\end{array}$ & $\begin{array}{c}\text { Viremia } \\
\text { Titer } \\
\left(\mathrm{LOG}_{10}\right. \\
\text { PFU/ml)* }\end{array}$ & Clinical Signs $* \dagger$ & $\begin{array}{l}\text { Final } \\
\text { Outcome }\end{array}$ \\
\hline $\begin{array}{c}\text { Control } 2 \\
1306064 \\
\text { (F) } 3.44 \mathrm{~kg}\end{array}$ & -5 & $\begin{array}{l}10.64(6) \\
11.45(8)\end{array}$ & $\begin{array}{l}7.83(6) \\
7.68(8)\end{array}$ & 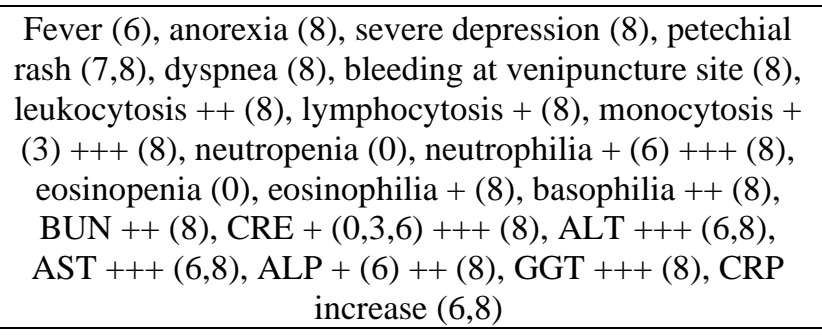 & $\begin{array}{l}\text { Euthanized } 8 \\
\text { DPI }\end{array}$ \\
\hline $\begin{array}{c}\text { Survivor } 6 \\
\text { Y1606047 } \\
\text { (M) } 3.50 \\
\text { kg }\end{array}$ & -5 & N.D. & N.D. & $\begin{array}{c}\text { Monocytosis }+(0,14), \text { neutrophilia }+(0), \text { eosinopenia } \\
(14,28), \text { basopenia }(28)\end{array}$ & Survived \\
\hline $\begin{array}{c}\text { Survivor } 7 \\
1309106 \\
\text { (F) } 3.20 \mathrm{~kg}\end{array}$ & -5 & N.D. & N.D. & $\begin{array}{c}\text { Monocytosis }+++(6), \text { neutrophilia }+(6), \text { eosinopenia }(0) \text {, } \\
\text { basopenia }(0)\end{array}$ & Survived \\
\hline $\begin{array}{c}\text { Survivor } 8 \\
1607197 \\
\text { (M) } 3.66 \\
\mathrm{~kg}\end{array}$ & -5 & N.D. & N.D. & $\begin{array}{c}\text { Monocytosis }+(3,6)++(0) \text {, eosinopenia }(6,10,21,28) \\
\text { eosinophilia }+(3) \text {, basopenia }(6,10,21)\end{array}$ & Survived \\
\hline $\begin{array}{c}\text { Fatal 1 } \\
1405226 \\
\text { (F) } 3.44 \mathrm{~kg}\end{array}$ & -5 & $\begin{array}{c}7.56(6), 11.88 \\
(9)\end{array}$ & $\begin{array}{l}4.02(6) \\
8.26(9)\end{array}$ & 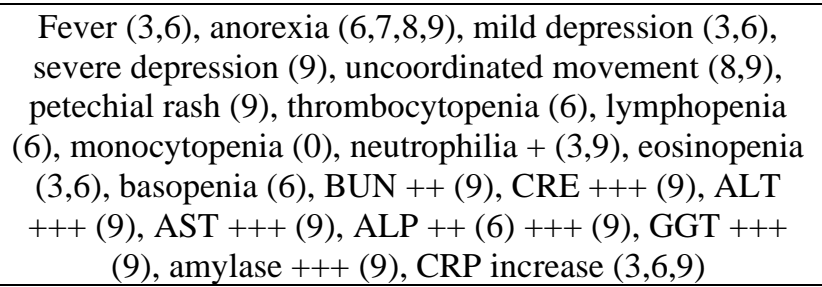 & $\begin{array}{l}\text { Euthanized } 9 \\
\text { DPI }\end{array}$ \\
\hline $\begin{array}{c}\text { Survivor } 9 \\
\text { Y1512011 } \\
\text { (M) } 3.32 \\
\text { kg }\end{array}$ & -5 & N.D. & N.D. & $\begin{array}{l}\text { Leukocytosis }+(3), \text { monocytosis }+(3,6,14) \text {, neutropenia } \\
\quad(28), \text { neutrophilia }+(3) \text {, eosinopenia }(6,21,28)\end{array}$ & Survived \\
\hline
\end{tabular}

951

952 S2 Table. Clinical findings in MARV-exposed cynomolgus macaques immunized with

\section{Vesiculovax vaccine 5 days prior to challenge.}


954 Macaques were immunized with a vector control (black; n=1) or rVSV-N4CT1-MARV-

955 GP vaccine at -5 DPI (brown; $n=5$ ). *Day after MARV challenge is in parentheses up to

956 the 28 DPI study endpoint. $\dagger$ Fever is defined as a temperature greater than $2.5^{\circ} \mathrm{F}$ above

957 baseline, at least $1.5^{\circ} \mathrm{F}$ above baseline and $\geq 103.5^{\circ} \mathrm{F}$, or $1.1{ }^{\circ} \mathrm{F}$ above baseline and $\geq$

$958104^{\circ} \mathrm{F}$. Leukopenia, thrombocytopenia, and lymphopenia are defined by a $>40 \%$ drop in

959 numbers of leukocytes, platelets, and lymphocytes, respectively. Leukocytosis,

960 monocytosis, and granulocytosis are defined as a $\geq$ two-fold increase in leukocytes,

961 monocytes, and granulocytes, respectively. Crosses indicate increases in liver enzymes

962 (ALT, AST, ALP, GGT) or renal function test values (BUN, CRE): 2- to 3-fold increase,

$963+$ + >3- up to 5-fold increase, ++; >5-fold increase, +++. Abbreviations: M, male; F, female;

964 kg, kilogram; PFU, plaque-forming units; MARV, Marburg virus; BUN, blood urea

965 nitrogen; CRE, creatinine; ALT, alanine aminotransferase; AST, aspartate

966 aminotransferase; ALP, alkaline phosphatase; GGT, gamma-glutamyltransferase; CRP, c-

967 reactive protein; DPI, days post infection. 
bioRxiv preprint doi: https://doi.org/10.1101/2022.01.22.477345; this version posted January 23, 2022. The copyright holder for this preprint (which was not certified by peer review) is the author/funder, who has granted bioRxiv a license to display the preprint in perpetuity. It is made available under aCC-BY-NC-ND 4.0 International license.

968

\begin{tabular}{|c|c|c|c|c|c|}
\hline $\begin{array}{l}\text { Animal ID } \\
\qquad(\text { sex }) \\
\text { weight }\end{array}$ & $\begin{array}{l}\text { Group } \\
\text { (Day of } \\
\text { Vaccination) }\end{array}$ & $\begin{array}{l}\text { RT-qPCR Titer } \\
\left(\mathrm{LOG}_{10}\right. \\
\text { copies } / \mathrm{ml})^{*}\end{array}$ & $\begin{array}{l}\text { Viremia } \\
\text { Titer } \\
\left(\mathrm{LOG}_{10}\right. \\
\text { PFU/ml)* }\end{array}$ & Clinical Signs $* \dagger$ & $\begin{array}{c}\text { Final } \\
\text { Outcome }\end{array}$ \\
\hline $\begin{array}{l}\text { Control } 3 \\
1508374 \\
\text { (F) } 2.96 \mathrm{~kg}\end{array}$ & -3 & $\begin{array}{l}11.70(6) \\
11.09(9)\end{array}$ & $\begin{array}{l}2.00(3) \\
8.33(6) \\
7.08(9)\end{array}$ & $\begin{array}{l}\text { Anorexia (8), mild depression }(8,9) \text {, petechial rash } \\
(6,7,8,9) \text {, diarrhea }(8,9) \text {, leukocytosis }++(9), \\
\text { thrombocytopenia }(9), \text { lymphopenia }(6), \text { lymphocytosis }++ \\
(9) \text {, monocytosis }+(3)+++(9), \text { neutrophilia }+(9), \\
\text { eosinopenia }(0,3,6) \text {, eosinophilia }+++(9) \text {, basopenia }(0,6) \text {, } \\
\text { basophilia }++(9) \text {, ALT }+++(6,9), \text { AST }+++(6,9), \text { ALP }+ \\
(9)++(6), \text { GGT }++(6,9), \text { CRP increase }(6)\end{array}$ & $\begin{array}{c}\text { Euthanized } \\
9 \text { DPI }\end{array}$ \\
\hline $\begin{array}{l}\text { Fatal } 2 \\
150820 \\
(\mathrm{M}) 6.80 \\
\mathrm{~kg}\end{array}$ & -3 & $9.52(6)$ & $\begin{array}{l}1.40(3) \\
5.98(6)\end{array}$ & $\begin{array}{l}\text { Fever (6), anorexia (6), petechial rash (6), bleeding nares } \\
\text { (6), thrombocytopenia (6), CRE ++ (6), ALT +++ (6), } \\
\text { AST +++ (6), ALP ++ (6), CRP increase (6) }\end{array}$ & $\begin{array}{c}\text { Euthanized } \\
6 \mathrm{DPI}\end{array}$ \\
\hline $\begin{array}{l}\text { Fatal } 3 \\
181131 \\
(\mathrm{M}) 3.00 \\
\quad \mathrm{~kg}\end{array}$ & -3 & $\begin{array}{c}5.58(3), 10.18 \\
(6)\end{array}$ & $\begin{array}{l}2.97(3) \\
6.34(6)\end{array}$ & $\begin{array}{l}\text { Fever }(3,6) \text {, anorexia }(5,6) \text {, moderate depression }(6), \\
\text { petechial rash }(6) \text {, rectal bleeding }(6) \text {, bleeding at } \\
\text { venipuncture sites }(6) \text {, thrombocytopenia }(6) \text {, lymphopenia } \\
(3), \text { monocytopenia }(0,3), \text { neutrophilia }+(3) \text {, eosinopenia } \\
(0,3,6) \text {, basopenia }(0,3,6), \text { BUN }+(6), \text { CRE }+(6), \text { ALT } \\
+++(6) \text {, AST }+++(6), \text { GGT }++(6), \text { CRP increase }(3,6)\end{array}$ & $\begin{array}{c}\text { Euthanized } \\
6 \mathrm{DPI}\end{array}$ \\
\hline $\begin{array}{l}\text { Fatal } 4 \\
140130(\mathrm{~F}) \\
2.84 \mathrm{~kg}\end{array}$ & -3 & $\begin{array}{l}6.00(3), 11.36 \\
(6), 9.23(7)\end{array}$ & $\begin{array}{l}3.78(3) \\
8.13(6) \\
8.16(7)\end{array}$ & $\begin{array}{c}\text { Anorexia }(5,6,7) \text {, severe depression }(7), \text { mild petechial } \\
\text { rash }(6,7) \text {, rectal bleeding }(7), \text { leukocytosis }+(7), \\
\text { lymphopenia }(0,3) \text {, lymphocytosis }+(7), \text { monocytosis }+ \\
(7), \text { neutrophilia }+(3,7) \text {, eosinopenia }(0,3,6) \text {, basopenia } \\
(3,6) \text {, basophilia }+(7), \text { ALT }+++(6), \text { AST }+++(6), \text { ALP } \\
++(6), \text { GGT }++(6), \text { CRP increase }(3,6)\end{array}$ & $\begin{array}{c}\text { Euthanized } \\
7 \text { DPI }\end{array}$ \\
\hline $\begin{array}{l}\text { Survivor } \\
10 \\
150966 \\
\text { (M) } 6.70 \\
\mathrm{~kg}\end{array}$ & -3 & $\begin{array}{c}6.76(6), 7.90 \\
(10), 5.48(14)\end{array}$ & $\begin{array}{l}2.89(3) \\
3.34(6) \\
4.26(10)\end{array}$ & $\begin{array}{l}\text { Anorexia }(6,7,8,9,10), \text { petechial rash }(9,10,11,12), \\
\text { leukocytosis }+(3,28)+++(14), \text { lymphopenia }(6), \\
\text { lymphocytosis }+(21)++(14), \text { monocytosis }+(3,21)++ \\
(14,28), \text { neutrophilia }+(6,10,28)++(3)+++(14), \\
\text { eosinopenia }(6), \text { eosinophilia }+(14)++(28), \text { basopenia } \\
(6), \text { basophilia }+(3,28)++(14), \text { BUN }++(10), \text { CRE }+ \\
(10), \text { ALT }+(21)+++(10,14), \text { AST }+(6)++(14)+++ \\
(10), \text { ALP }+(6)++(28)+++(10,14,21), \text { GGT }+(28)++ \\
21)+++(10,14), \text { CRP increase }(6,10)\end{array}$ & Survived \\
\hline $\begin{array}{c}\text { Fatal } 5 \\
\text { Q1502038 } \\
\text { (F) } 3.24 \mathrm{~kg}\end{array}$ & -3 & $\begin{array}{l}5.05(3), 10.22 \\
\quad(6), 8.62(7)\end{array}$ & $\begin{array}{l}2.44(3), \\
8.49(6), \\
7.32(7)\end{array}$ & $\begin{array}{l}\text { Anorexia }(6,7), \text { petechial rash }(6,7), \text { dyspnea }(7), \\
\text { thrombocytopenia }(6,7), \text { lymphopenia }(3,6), \text { neutrophilia }+ \\
(3,7), \text { eosinophilia }+(3)++(7), \text { BUN }+(6)+++(7), \text { CRE } \\
++(6), \text { ALT }+++(6,7), \text { AST }+++(6,7), \text { ALP }+++(6,7), \\
\text { GGT +++ }(6,7), \text { amylase }+(7), \text { CRP increase }(3,6,7)\end{array}$ & $\begin{array}{c}\text { Euthanized } \\
7 \text { DPI }\end{array}$ \\
\hline
\end{tabular}

969

970 S3 Table. Clinical findings in MARV-exposed cynomolgus macaques immunized with

\section{Vesiculovax vaccine 3 days prior to challenge.}

972 Macaques were immunized with a vector control (black; $n=1$ ) or rVSV-N4CT1-MARV-

973 GP vaccine at -3DPI (red; $n=5$ ). *Day after MARV challenge is in parentheses up to the 
97428 DPI study endpoint. †Fever is defined as a temperature greater than $2.5^{\circ} \mathrm{F}$ above

975 baseline, at least $1.5^{\circ} \mathrm{F}$ above baseline and $\geq 103.5^{\circ} \mathrm{F}$, or $1.1^{\circ} \mathrm{F}$ above baseline and $\geq$

$976104^{\circ} \mathrm{F}$. Leukopenia, thrombocytopenia, and lymphopenia are defined by a $>40 \%$ drop in

977 numbers of leukocytes, platelets, and lymphocytes, respectively. Leukocytosis,

978 monocytosis, and granulocytosis are defined as a $\geq$ two-fold increase in leukocytes,

979 monocytes, and granulocytes, respectively. Crosses indicate increases in liver enzymes

980 (ALT, AST, ALP, GGT) or renal function test values (BUN, CRE): 2- to 3-fold increase,

$981+$ +; >3- up to 5-fold increase, ++; >5-fold increase, +++. Abbreviations: M, male; F, female;

982 kg, kilogram; PFU, plaque-forming units; MARV, Marburg virus; BUN, blood urea

983 nitrogen; CRE, creatinine; ALT, alanine aminotransferase; AST, aspartate

984 aminotransferase; ALP, alkaline phosphatase; GGT, gamma-glutamyltransferase; CRP, c-

985 reactive protein; DPI, days post infection.

986

\section{Funding}

988 This study was supported in part by the Department of Health and Human Services,

989 National Institutes of Health, grant number U19AI142785 to TWG. Operations support of

990 the Galveston National Laboratory was supported by NIAID/NIH grant UC7AI094660.

991

992 Availability of data and materials

993 The datasets used and/or analyzed during the current study are available from the 994 corresponding author upon request. 


\section{Author contributions}

997 TWG, MAE, JHE, and DM conceived and designed the study. CG, TEL, and DM designed

998 the vaccine vectors and did preparative work. DJD, JBG, and TWG performed the

999 challenge experiments. CW, RWC, DJD, JBG, and TWG performed animal procedures

1000 and clinical observations. VB and KNA performed the clinical pathology assays. VB

1001 performed the plaque assays. KNA performed the PCR assays. CW performed the

1002 transcriptomic assays and bioinformatics. CW performed the ELISAs. KAF performed the

1003 necropsies and gross pathology analysis. All authors analyzed the data. CW wrote the

1004 paper. RWC, TWG, and DM edited the paper. All authors had access to the data and

1005 approved the final version of the manuscript. 